\title{
Chorology of the European hypogeous Ascomycetes, I.
}

\author{
Elaphomycetales
}

\author{
MARIA LAWRYNOWICZ
}

Laboratory of Mycology, Institute of Environmental Biology, Lodż University, S. Banacha 12/16, PL-90-237 lódż, Poland

Law rynowicz M.: Chorology of the European hypogeous Ascomycetes, I. Elaphomyceiales. Acta Mycologica 25(1): 3-41. 1989.

Distribution of hypogeous Asconyceles in Poland with regard to their areals in Europe is discussed in the paper. The results are illustrated on 63 maps. The paper is the second part of a monographic study of the Elaphomycetales and Tuherales worked out by the author in the Polish Flora - Mycota (rol. 18, 1988).

1. Introduction

2. State of investigation on chorology of hypogeous Ascomycetes

3. Material and methods

4. Comments on text and maps

5. Chorology of species of Elaphomycetales

5.1. Elaphomyces gramdatus $\mathrm{Fr}_{\mathrm{r}}$ emend. Holl.

5.11. E. virgatosporus Holl.

5.2. E. asperulus Vitt.

5.12, E. moreltii Vitt.

5.13. E. aculeatus Vitt.

5.4. E. papillanus Vitt

5.14. E. echinatus Vitt.

5.5. E. atropurpureus Vitt.

5.15. E. cyanosporus Tul.

5,6. E. leucospories Vitt.

5.16. $\boldsymbol{E}$. foetidus Vitt.

5.7. E. maculates Vitt.

5.17. E. persoonii Vitt.

5.8. E. leveillei Tul.

5.18. E, mutabitis Vitt.

5.9. E. anthracimus Vitt.

5.19. E. cirrinus Vitt.

5.20. E. striatospanis Kers

5.10. E. septatus Vitt.

6. Chorology of Cenococcum geophilum Fr. (Deuteromycetes) 


\section{INTRODUCTION}

Knowledge of the geographical distribution for species of hypogeous fungi, and of their local frequency, is important in the understanding of their living requirements. An analysis of herbarium material concerning the area under investigation helps us in the correct interpretation of changes within a species. This is especially valuable when dealing with either extreme forms or deformed specimens resulting from habitat differences and, indirectly, from the geographical situation. This is why the author's taxonomic studies on hypogeous ascomycetes (Lawrynowicz 1988) refer to the chorological analysis.

The present account takes into consideration both the author's collections, gathered over 12 years or so, and the available herbarium material from the whole of Europe, with the following aims: (a) to gather all available information on the appearance of those species of hypogeous ascomycetes which may extend into parts of Poland; (b) to represent cartographically the current investigative state of these fungi both within Poland and Europe generally; (c) to interpret their geographical distribution and attempt to determine the hypothetical range of particular species within Europe.

All the material collected by the author, together with a card-index of the analysed specimens, is deposited at the Laboratory of Mycology, Institute of Environmuntal Biology. University of Lódz.

\section{STATE OF INVESTIGATION ON CHOROLOGY OF HYPOGEOUS ASCOMYCETES}

In comparison with the knowledge on higher plant geography, for which maps and distribution atlases are available, the chorology of hypogeous fungi, especially Ascomycetes, is poorly understood. The early investigators ignored distribution but concentrated on the taxonomic problems. More recent authors (Knapp, 1950-52; Hawker 1954; M. Lange 1956: Eckblad 1954, 1962; Szemere 1965; De Vries 1971; Kers 1978, 1979a, 1979b, $1980,1983)$ include both locality indices and exsiccata citations. Recently, Kers (1983) included in his paper a distribution map of Elaphomyces anthracimus and $E$. leveillei in Scandinavia, prepared on the basis of a critical revision of both herbarium material and his own collections.

Attempts to establish distribution schemes for some Agaricales have appeared in the literature (B is by 1933; Vasilk ov 1955; Vasileva 1967. 1973; L. Lange 1974; Wasser 1980), indicating some generalizations which could possibly be applied to hypogeous fungi. By analogy to higher plants, these authors attempted to establish range types, concluding that the mycoflora was changing much more in a north-south direction than east-west (Vasilkov 1955). On the other hand the ranges cxhibited by fungi are larger 
than those shown for higher plants (Vasilkov 1955; Ainsworth 1971). V asileva $(1967,1973)$ proposed a revised classification, specifically for fungi, recognising ten range types. These papers on Agaricales emphasise the ease of air-dispersal of spores over wide areas, and of the possibility of substratum and mycelial disturbance.

Such criteria, however, do not apply to hypogeous fungi. They cannot release spores either from the asci or from the fruitbody, and spores are therefore not wind disseminated. Most species are mycorrhizal, hence the concept of Raitwiir (1964) should be followed with the starting point for mycogeographical analysis and classification based on the individual range of fungus species. In fact, hypogeous species offer excellent material for the determination of individual ranges of fungi.

Many of the important collections from the last century do not include locality details. However, the early monographs (Vittadini 1831, 1842; Berkeley, Broome 1844; Tulasne 1851; Zobel 1854) as well as later ones (Chatin 1892; Hesse 1894; Fischer 1897; Schroeter 1908; Bucholtz 1902; Hollos 1911) attempt to generalise on the distribution by indicating countries and geographical areas for the species described. The published distribution maps cite only a few references, although the economic importance of edible truffles made them an exception. These latter maps were based on truffle localities related to the commercial sale in Mediterranean countries (Nicolas 1975; Delmas 1978).

The exploration of hypogeous fungi prior to World War 1 in central Europe was limited to the discovery of whether or not edible truffles existed and where they could be found. Numerous records were published, including some from northern Poland and from Silesia, which were analysed by Hesse (1894) and Schroeter (1908) who concluded that only a few were valid. Eichler (1904) reported the occurrence of Elaphomyces, Hydnotrya and Tuher. A comprehensive reference list dealing with the state of investigation within Polish territories was provided by the present author (Lawrynowicz 1988) dealing with Elaphomycetales and Tuberales.

The earliest data recorded by map for hypogeous fungi in Poland was published by Bucholtz (1902). In a map of Russia, he indicated a locality on the Vistula river, close to Warsaw. Between the two world wars, Teodorowicz $(1928,1933,1936)$ undertook an intensive search for underground fungi in Pomerania, Southern Poland and Great Poland, and his collection of Tuberales proved to be of especial interest.

The first paper of a chorological character was published by Lubelsk a (1953), presenting the occurrence in Poland of Choiromyces meandriformis and eight taxa of Tuber taken from the available literature. This paper summarised all the available data and represents a starting point for hypogeous fungi investigation in Poland. 
Taking into account both the published data and a critical revision of the herbarium material, Skirgiełto \& Wosinska (1963) published maps of the distribution of localities for four species of Elaphomyces in Poland, and Eawrynowicz (1970) provided some additional localities from Central Poland.

From a chorological standpoint, Choiromyces meandriformis is one of the best known species for Europe. Distribution has been described by Skirgiełto (1976); Gross (1977); Kreisel, Dörfelt \& Benkert (1980); and Babos (1981). In the same paper, Babos also provided the distribution of Terfezia terfezioides within Hungary.

Recent monographic accounts have become increasingly orientated towards taxonomic-chorological studies of individual species.

\section{MATERIAL AND METHODS}

The present account, as far as distribution in Poland and the rest of Europe is concerned, is based upon herbarium studies, and only personally cxamined exsiccata have been concerned. In a few well documented cases only (e.g. Hawker 1954; Kers 1983), have literature data been mapped by the present author.

The number of exsiccata extending back to the middle of the last century is apparently quite large. In many cases, however, the collections found in various herbaria represent only a few localities. There was extensive interchange of material between the early collectors, and even small collections were divided into many portions. It is not unusual to find on the sheets in various herbaria only a single specimen or even a small part of one fruitbody coming from the same locality. For parts of Europe where there has been no intensive collecting or investigation the number of known localities remains very small, even for the common species.

The herbarium material studied includes all collections available both within and outside the country under investigation, within Europe. About 1500 collections of the author's own material has been studied, together with about 5000 sheets from 44 herbaria. The list of herbaria and the material examined is given in the taxonomic part, along with keys, descriptions and illustrations to 81 species of Elaphomycetales and Tuberales (Lawry nowicz 1988). When finalising this list, a considerable amount of examined material had to be omitted owing to an unclear taxonomic interpretation, the unlikely occurrence in Poland, the poor state of preservation of the material, or the quality of the material (fruitbodies too young or too old). Quite often a good specimen could not be utilised for the chorological part of the work because the collection details were absent, illegible or unclear in meaning. The 
intention has been to use only material accurately identified and accompanied with precise locality details. Collections gathered by the present author form the basis for the distribution maps of hypogeous fungi in Poland, supplemented by other collections coming from Polish and some overseas herbaria.

An initial analysis revealed the majority of records came from areas which had proved geobotanically attractive to naturalists. For this reason, field investigations were directed toward areas and habitats which had been previously ignored, such as Central Poland. Polish exsiccate material came from: Kraków herbaria (KRA - 29 sheets, KRAM - 48 sheets), mainly from Southern Poland, collected by K. Rouppert, B. Gumińska, W. Wojewoda, et al.; the Warsaw herbarium (WA - 23 sheets), from the Tatra Mtns, and the environs of Warsaw, collected by A. Skirgiello, W. Rudnicka-Jezierska, S. Frejlak, et al.; Lublin herbarium (LBL 20 sheets), from the Lublin Upland and heights of Roztocze, collected by B. Sałata: Poznań herbarium (POZ -2 sheets), from the Baltic coast, collected by M. Lisiewska; Wroclaw herbarium (WRSL - 60 sheets), from the Sudetes and Silesia (in herb. Schroeter, but owing to illegibility of labels only $60 \%$ i.e. 32 sheets could be considered); Łódź herbarium (LOD - ca 1500 sheets).

The majority of overseas collections date comes from the last century. The earliest exsiccate collection is dated 1839, and is deposited in the Bratislava museum. The collections of Saccardo in Padua (PAD), Tulas ne \& Tulasne in Paris (PC), and Berkeley \& Broome in Kew (K), contain exsiccata either exchanged or donated e.g. by Vittadini, may be earlier but are undated. Many of the collections include important mycothecas, which are to be found in several of the older herbaria e.g. Thumen, Mycotheca universalis; Saccardo, Mycotheca italica; Sydow, Mycotheca germanica, etc. Within Europe, the Munich herbarium (M) houses the richest and well preserved collection, containing the central European collections, including both the older material and well documented contemporary material e.g. E. Soehner and G. Gross. Kew (K) also houses very rich collections, including overseas material, and in recent years has acquired the collections of L. Hawker. The many type collections also make the Paris herbarium (PC) an important source, with many collections from the last century. In central Europe, the important herbaria include Budapest (BP), with the collections of L. Hollos and L. Szemere, and Prague (PRM), which houses both Czechoslovakian material and also old and new material from overseas.

Especially significant are the Scandinavian herbaria: Copenhagen (C). with the Danish collections revised by $\mathbf{M}$. L a nge according to contemporary criteria, and Oslo $(\mathrm{O})$ containing Norwegian material collected and examined 
by F.-E. Eckblad. In Finland, the collections in Helsinki ( $\mathbf{H}$, HFR), Turku (TUR), and Oulu (OULU), include abundant material of Elaphomyces, both the older collection of Hittonen and more recent material by E. Ohenoja and T. Ulvinen. The Swedish herbaria of Stockholm (S) and Uppsala (U) also represent some of the richest collections in Europe, with the Swedish collections of Elaphomyces and, in addition. Tuberales material from several European countries, with both older collections from the last century and more recent specimens e.g. due to L. E. Kers. The collection at Lciden (LD), recently revised by De Vries, must also be taken into account. From eastern Europe, the Leningrad herbarium (LE) has been analysed, but of the mategial available to the author, only a few collections originated from within Soviet territory, the majority coming from western Europe by exchange. Collections from the Iberian Peninsula have also been analysed: Madrid herbarium (MAF), includes the collections of F. D.C Calonge, and the Lisbon herbarium (LISU) houses the Pinto-Lopez collection. Since the majority of the latter specimens belong to Terfeziaceae, the collections also illustrate, to some extent, the flora of the north coast of Africa and Asia Minor.

The entire material, analysed microscopically, is arranged according to species locality accounts, and provides the documentation for the species distribution maps. The localities in Poland are shown on maps with a 10 $\times 10 \mathrm{~km}$ squares grid. As a result of detailed examination, a square on the map may include several localities so, for reasons of completeness, the list of localities found is appended, with the use of abbreviations explained below. The order of localities corresponds to the physico-geographical partition of Poland according to Kondracki (1977). The background grid used for the maps of Poland was originally used in the "Atlas of Vascular Plant Distribution in Poland", ATPOL (Zając 1978). The meridian 19 E is the grid axis, the grid being marked on the Poland map 1:1000000. All localities shown on the map were previously identified on detailed topographical maps. The area of Poland is covered by 3437 squares. The cartograms used in this account are in accordance with those adopted by the authors of ATPOL.

Collections from other European countries are used to determine the overall distribution of individual species by the point method, whilst aggregation of the points are indicated by cross-hatching. The species locality accounts form an integral part of that section of the account, and each record corresponds to a herbarium label. For purposes of recording the distribution, data from the literature were utilized where they are important for species chorology, but these localities are not marked on the maps.

The maps presented provide only an introduction to fungal chorology, however, even such a limited account indicates the need and effectiveness in searching for hypogeous fungi in specific sites. 


\section{COMMENTS ON TEXT AND MAPS}

The chorological characteristics of the species are arranged systematically according to the taxonomic part of the investigation (Lawrynowicz 1988). Well known species have separate subsections, whilst the others are treated collectively within the genus and may therefore occasionally diverge from the systematic order. Similarly species with few known localities may appear on the same map.

Localitics are defined as a point on the terrain whose distance from the next nearest point (place of appearance of the species) is not less than $1 \mathrm{~km}$ in the lowlands, and $0.5 \mathrm{~km}$ in the mountains. These localities are arranged according to the macroregions proposed by Kondracki (1977). Within a macroregion. the arrangement is alphabctical and the number of squares on the map is provided.

The locality description includes:

a - name of macroregion (ace, Kondracki 1977):

b - map coordinates according to the formula ATPOL i.e, first letter and second numeral correspond to column on map, whilst the second letter and first numeral correspond to the strip, e.g. AB 23 indicates strip B2, column A3; the coordinates appear on map-frame;

c - name of nearest point, reserve, forest range, forest inspectorate, or two points between which the locality occurs, or the name of the mountain. hill, valley, etc.;

d - names of trees, shrubs under/at which the fungus has grown fabbreviations used in forestry, see Trampler, Smykata \& Bosiak 1960), finally the plant community abbreviation:

e - date (month and year) of collection:

$f$ - name or initials of collector:

g - herbarium abbreviation (Index Herbariorum 1981) housing collection, together with herbarium specimen number.

The locality descriptions adopx the following punctuation:

. full stop - completes macroregion description,

: semi-colon - separates successive localities in a mezoregion.

, comma - scparates sucoessive localities,

- dash - precedes locality description for subsequent square.

Localities are eited within the list in such a way as to allow the reader to find them on the map. To find the locality in the country, the reader must refer to the species locality accounts for further details.

List of abbreviations

Abbreviations of names of trees, shrubs and plant communities
Aa - Abies alba
Ag - Alnus glutinosa
Ap - Acer plananoides
Aps - Acer pseudoplatamis
$B p p-B e t u l a$ pendula and $B$. pubescens
$\mathrm{Ca}-$ Corylus avellaria

$\mathrm{Cb}$ - Carpinus betulus

$\mathrm{Fe}$ - Fraxinus excelsior

$F Q$ - Fago-Quercetwn

Fs - Fagus syltatica

Jc - Juniperus communis

Ldp - Larix decidua and L polonica 

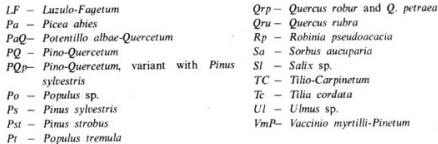

Abbreviations of collectors names
AS - Alina Skirgiello
BG - Barbara Guminska
BS - Bogustaw Salata
KM - Kazimierz Mamos

$$
\begin{aligned}
& \text { MK - Maria Kurzac } \\
& \text { Mt - Maria Lawrynowicz } \\
& \text { RO - Romuald Olaczek } \\
& \text { WW- Wladyslaw Wojewoda }
\end{aligned}
$$

Other abbreviations

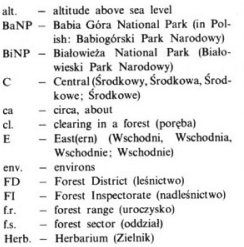

$$
\begin{aligned}
& \text { Mt - Mountain (Góra) } \\
& \text { Mtns - Mountains (Góry) } \\
& \text { N - North(crn) (Północny, Północna, } \\
& \text { Północne; Pólnocne) } \\
& \text { n. }- \text { near } \\
& \text { OjNP - Ojcow National Park (Ojcowski } \\
& \text { Park Narodowy) } \\
& \text { prov. - province (in Poland: województwo) } \\
& \text { Res. - Reserve (rezerwat) } \\
& \text { S - South (ern) (Poludniowy, Poludnio- } \\
& \text { wa, Poludniowe; Poludniowe) } \\
& \text { Str. - Street (ulica) } \\
& \text { W - West(ern) (Zachodni, Zachodnia, Za- } \\
& \text { chodnie: Zachodnic) } \\
& \text { WoN - P Wolin National Park (Wolinski } \\
& \text { Park Narodowy) }
\end{aligned}
$$

\section{CHOROLOGY OF SPECIES OF ELAPHOMYCES}

5.1. Elaphomyces granulatus Fr, emend. Holl.

Distribution - in Europe (Fig. 1). The species is frequently found in Central Europe and southern Scandinavia in lowlands and low mountain sites. It also occurs in Great Britain (England, Wales, Scotland), southern France, Belgium, The Netherlands, and in European USSR. Northern localities extend to the arctic circle in Finland and Norway; the northernmost site 


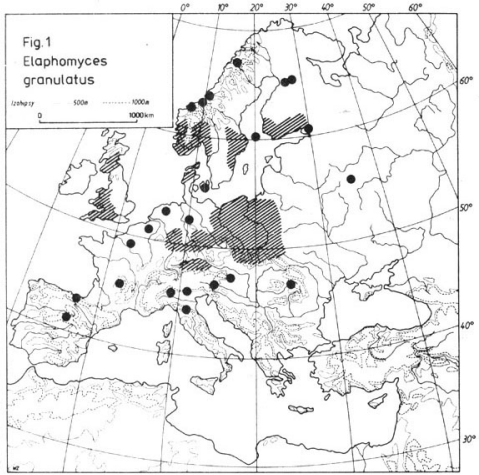

is at Leirford, Nordland prov, at $1000 \mathrm{~m}$ alt. It occurs in the Carpathian Mountains, especially frequent in the Tatra and Beskid Mtns, more rarely in Transylvania. In southern Europe, it occurs mostly in the uplands and mountains, along the southern slopes of the Alps (Cavelonte, environs of Turin, Klagenfurt), Vallombrosa, the Central Massif, and the mountains of the Iberian Peninsula). The highest recorded localities are Važec (Tatra Mtns) at $850 \mathrm{~m}$ alt. Vallombrosa (Appenine Mtns) at ca $1000 \mathrm{~m}$ alt., and environs of Klagenfurt (Alps) at ca $800 \mathrm{~m}$ alt.

Hypothetical distribution. All Europe, within borders of nemoral and boreal zones (deciduous, mixed and coniferous forests), and analogous mountainous sites. Absent from Steppe and Mediterranean vegetation areas. Most common in region of oceanic climate. 
Habitat. A typical forest species, forming ectotrophic mycorrhizal associations probably with several tree species. Found in deciduous and mixed tree stands, more rarely in coniferous stands. According to some authors Fagus syluatica is the principal mycorrhizal associate, but in its absence will form associations with other deciduous and coniferous trees e.g. Picea abies in Norway. Known in Poland from 121 localities. Often occurring with $E$. muricatus, more rarely with $E$. asperulus but (in comparison with the latter) in lower mountain sites. Prefers rather fertile soils, with $\mathrm{pH} \mathrm{4-5.} \mathrm{Other}$ accompanying species: Genea hispidula, Hydnotrya tulasnei, and the sclerotia of Cenococcum geophilum. The fruitbodies are sometimes attacked by Cordyceps capitata and $C$. ophioglossoides.

Distribution in Poland (Fig. 2). Occurring throughout the entire country, including coast, lakelands, uplands and mountains. One of the most common species in the mycoflora.

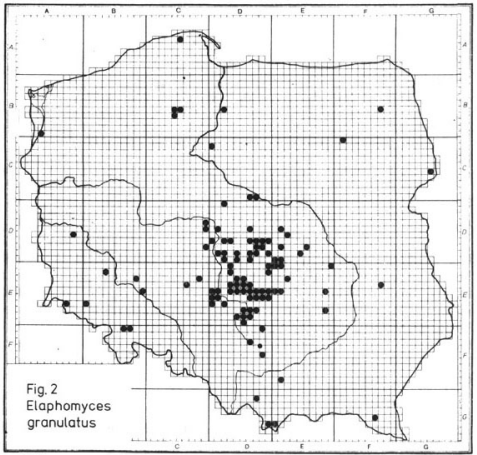

sem - . 
Localities in Poland. Pobrzeże Baltyckie - AB 93 Puszca Bukowa Res. Bukowe Zdrojc. Fs Pa Ps Cb Qrp. 06.74: Mł, LOD 20179 \& 20246; - CA 45 Choczewo. Pa, 07.82: MŁ LOD 20790, Pojezierze Południowo pomorskie - CB 54 Bory Tucholskic Konigort - Klodawa, Ps Bpp, 07.73: Mt, LOD 20318; - CB 55 Zukowo, Ps, 07 \& 08.74: M1, LOD 20445 \& 20447; - CB 64 Raciąski Miyn, Ps cl., 07.73: Mt, LOD 20446. Dolina Dolnej Wisly - DB 52 FD Bialki n. Kwidzyn, PaQ, 04.76: T, Zaluski, LOD 20224. Pojezierze Chełmińsko-Dobrzyniskie - DC 10 env. of Chelmźa, Ps, 06.80: A. tawrynowicz, LOD 20187. Pradolina Toruásko-Eberswaldzka - DC 96 Lucień n. Gostynin, Ps, 07.79. RO, LOD 20192. - Lack TC Ps Cb Qrp. 07.79: RO, LOD 20184 \& 20791. Pojezicrze Wielk opolskic - DD 02 Kujawy fr. Rogożno, Qrp Sa, 09,79: RO, LOD 20494. Wzniesienia Zielonegórskie - AD 58 Zielona Góra, Ps, 08,74: MŁ. LOD 20449. Niziny Sasko-kuźyckie - BE 13 Lubin, ImP, 06.73: WW, KRAM 14872. Nizina Poludniowowielkopolska - CD 39 Dąbrowice n. Kolo, Qrp Ps, 10.78: ML, LOD 20185: - CD 49 Małosryna n. Turek, Ps Qrp, 10.78: MŁ, LOD 20186; - CD 79 Sokołów n. Kożminek, Ul Rp Qrp Fs, 10.78: RO, LOD 20238; - CE 28 Res. Rys n. Lututów, Fs Aa Pa. 07.79 \& 09.80: ME \& RO, LOD 20223 \& 20501; - CE 36 Ustronic n. Kepno, Ps, 01.80: RO. LOD 20783: - DD 41 f.r. Wielenin n. Uniejów, Qrp Cb Ps, 10.78: Mt, LOD 20183; - DD 60 Linne n. Dobra, Ps Qrp, 10.78: ML, LOD 20230 \& 20237; - DD 62 Zakrzew n. Poddębice, Qrp Ps Pi, 05.80: KM. LOD 20453 \& 20455; - DD 63 Sarnów, Ps Qrp. 06.82: H. Andrzejewski, LOD 20771, - env. of Res. Jodly Oleśnickie, Ps, $07.82:$ KM \& MK, LOD 20772-73; - DD 81 Rossoszyca, $P_{s}$, 04.75: M1, LOD 20370; - DD $82 \mathrm{fr}$. Wojstawice n. Szadek, Pa Bpp, 1081: MK, LOD 20770; - DD 84 Jasionna n. Lask. Ps Qrp Bpp Pa. 04.75: RO, LOD 20415; - DD 92 Res. Jablecrnik n. Zduriska Wola, Qrp, 07.76: KM. LOD 20225; - DD 94 f.r. Ostrów n. Lask. Ps Qrp, 06.79: RO. LOD 20457 \& 20495; - DE 03 FI Sedriejowice, Ps, 11.73: M1. LOD 20402; - DE 24 f.r. Slupia n. Belchatow, Ps $P_{i} P_{a} J_{c}, 06,80$; RO \& L. Samosicj, LOD 20175 \& 20191: - DE 25 Nowy Świat, Ps Qrp Bpp, 06.80: RO, LOD 20174; - DE 33 f.r. Stróża n. Szezerców, Ps, 06.80: L. Samosiej, LOD 20178 ; - DE 34 f.r. Wola Wydrzyna, Qrp, 06.79: E. Filipiak, LOD 20220: - DE 35 Huba Ruszczyńska n. Szczerców, Ps Qrp cl, 08.78: RO \& MŁ, LOD 20236, 20452 \& 20491: - DE 36 f.r. Pytlowice FI Kamieńsk, Ps Cb Pa, 08.78: Mt, LOD 20241. Nizina Śląska - BE 28 Oborniki Slaskic. Herb. Schroct. WRSL; - CF 23 Biala n. Prudnik, Herb. Rehm S: - CF 28 Widow, Ps, 03.13: Herb. Sydow S \& Herb. Saccardo PAD. Nizina Pólnocnomazowiecka - FC 01 Kuzie n. Lomża, Ps, 03.76: P. Genow, LOD 20229.

Nizina Środkowomazowiecka - DD $46 \mathrm{fr}$. Bielawy - Mroga, Cb, 10.78: Mh, LOD 20400; - ED 41 Puszcza Bolimowska f.r. Bolimów, TC PaQ Ps Cb Bpp Qrp. 05.82: KM \& MK, LOD 20737-38; - ED 52 fr. Pusecza Mariańska, Ps Qrp, 05.82: MK, R. Dąbski, KM \& E. Wiącek, LOD 20739-54. Wzniesienia Poludniowomazowieckic - DD 66 Łódź Las Łagiewniki, Qrp Fs Cb Pa Ps, 01-10.74; Mt, LOD 20207-13, 20216-17, 20406-07 \& 20422, 05.77: KM \& L. Samosicj, LOD 20287 \& 20371; - DD 67 f.r. Janinów n. Brzcziny, Ps Fs. 11.73: Mt, LOD 20412, 10.78: E. Chalcarz, LOD 20188; - DD 68 Syberia n. Brzeziny, Ps, 04.74; RO, LOD 20366; - DD 69 f.r. Gorki n. Rogów, Ps, 05.74: RO, LOD 20369, - Res. Doliska n. Rogow, $A a$ Qrp Cb Pa PS, 05.74: RO, LOD 20763; - DD 77 f.r. Wiąezyñ n. Nowosolna, Ps Fs. Pa Pi, 07.74 \& 05-11.75; KM, A. Wiśniewska \& Z. lawnicka, LOD 2021415, 20233, 20242-43, 20312-16, 20319-21, 20404, 20417-19 \& 20755.57; - DD 78 Tadjin n. Brzeriny, PaQ. 05.74: RO. LOD 20367; - DD 86 Łódź Starorudzka Str. Ps, 04.79: KM. LOD 20244, - Łodź Las Komunalny - Skrajna, Ps, 04.79: KM, LOD 20245, - Lódź Zakładowa Str., PS, 11.78: KM, LOD 20190; - DD 87 Justynów n. Lodź, Qrp Ps Bpp Fs Cb, 08.80: A. Ławrynowicz, LOD 20239, 08.82: L. Samosiej, LOD 20761-62, - Janówka n. Justynów, PS Cb Fs, 08.82: L. Samosiej, LOD 20758-60; - DD 89 Budziszewice n. Koluszki, Ps 11.74: A. Olaczek, LOD 20403; - DD 96 Modica n. Lods, Ps, 03.80: J. Adamezyk \& KM, LOD 20232, - Tuszynck n. Łódź, Ps, 03.77: M. Borowska, LOD 20189, - Res. Molenda, Qrp Ps, 
10.79: ML, LOD 20416, - Res, Wolbórka n. Lódź, Ps Qrp. 05.74: ML LOD 20450; - DE 09 env, of Tomaszów Mazowiecki, Ps, 11.77: ML, LOD 20414; - DE 19 Res, Lubiaszów, Aa Cb. 08.72: ML, LOD 20766, 09.72: B. Rzerzycha; LOD 20365; - DE 44 Pickary n. Sulmierzyce, Ps Qrp, 05.79: RO, LOD 20456 \& 20498; - ED 71 Podlas n. Rawa Mazowiecka, Qrp Ps, 08.81: RO. LOD 20500, 20503-05 \& 20767-68; - ED 75 env. of Mogielnica n. Grojec, Ps Ca, 09.79: KM, LOD 20424; - ED 84 Res. Tomczyce n. Nowe Miasto, Ps Qrp Jc, 07.74: Mł. LOD 20361: - ED 90 Dąbrówka FI Lubochnia, Ps, 08.81: RO, LOD 20499, - f.r. Cygan n. Tomaszów Mazowiecki, Ps el., 06.80: ML, LOD 20177 \& 20764; - ED 91 Spala, Ps, 05.77: ML LOD 20362, 20408 \& 20372, - Teofilow n. Inowlódz, Qrp Pl, O8.78: ML, LOD 20492; EE 00 Borki n. Spala, Ps Qrp, 11.73: Mk, LOD 20411: - EE 01 Inowlodz, Ps, 05.77: Mt. LOD 20363. Przedgórze Sudeckie - BE 86 Dzierzoniów, 05.1861: Herb. Schroet. WRSL: - BF 07 Las Sieroszów n. Ząhkowice Sląskie, 06.17: M. Sudety Zachodnie - AE 67 Górzec. 05.1889: Herb. Schroet. WRSL: - AE 67 Świeratów, 05.1888: Herb. Schroct. WRSL: - BE 60 Szklarska Porçba. 08.1882: Herb. Schroct. WRSL. Sudety Srodkowe - BF 06 Srebrna Góra - Zdanów, 10.24: M. Wyżyna Woźnicko-Wicluáska - DE 40 f.r. Bukowce n. Zalęcze, Ps, 07.79: ML \& RO, LOD 20218-19 \& 20496, - Kamionka FI Mierzyce, Ps Qrp $P a, 04.75:$ MK, LOD 20364, - f.r. Mierzyce, Ps Qrp Pa, 07.82: MK, LOD 20778.82: - DE 41 env, of Krzezów, Ps, 08.79: K Cyžewska. LOD 20308, - f. Nizankowice, Ps, 09.79: Mt, LOD 20176, Ps Fs Qrp. 05 \& 08.82: MK. LOD 20774-77; - DE 43 Res. Murowaniec n. Pajeczno, Aa, 04.79: RO, LOD 20458; - DE 50 Zatecce Male, Ps Qrp, 07.79: ML. LOD 20497: - DE 51 Res. Węże, Ps Bpp Jc, 07.79: RO, LOD 20451: - DE 60 Zajaczki n. Kracpice, Fs Aa. 04.74: Mh LOD 20448; - DE 62 Miedzno n. Klobuck. Ps Qrp. 09.79: Mf. LOD 20307 \& 20309-10. Wyzyna Krakowsko-Częstochowska - DE 84 Mstów n. Częstochowa, Fs $B p p, 08.81$ : RO, LOD 20231 \& 20235; - DE 85 Smiertny Dąb FI Zloty Potok, Ps Pa, 04.75 ML LOD 20311: - DE 95 Potok Zloty, Fs, 0982: KM, LOD 20787; - DF 08 Oludza F1 Szczekociny, Qrp Ps, 09.82: KM, LOD 20788: - DF 26 Rodaki n. Ogrodzicniec, Fs, 05.80: Mt. LOD 20506; - DF 48 OjNP Ojeów - Skala ("Miotelka"). PQ, 03, 04 \& 09.61: WW, KRAM 13110 \& 13107-08, PQP, 03.61, 06.62 \& 63: WW, KRAM 13109 \& 13111-12, - Ojoów Smardzewice, PQP, 03.61: WW, KRAM. Wyzyna Przedborska - DE 45 Hulanka n. Dobryszyce, Ps Pa Qrp Jc Ca Bpp, 09.78: RO, LOD 20454; - DE 46 Kletnia, Ps Qrp, 0879 : Mt LOD 20180; - DE 47 Kodrąb, Ps, 08.79: M Ł, LOD 20181; - DE 48 Bakowa Gora, Ps Qrp cl. 05.80; RO, LOD 20765: - DE 49 f.r. Wilkowice, Ps Cb, 07.81: RO. LOD 20502; - DE 56 Przybysców n. Kobicie Wielkie, Ps Qrp Cb Tc Sa, 09.78: RO, LOD 20182, - Res Jasień, Ps Pa, 05.75: Mł, LOD 20420-21; - DE 57 f.r. Modrzewszexyzna n. Kobicle Wielkie, PaQ, 09.78: RO, LOD 20401; - DE 58 Mt. Chelmo, Qrp Cb Pt Pa, 08.79: Mt, LOD 20221 \& 20789; DE 59 env. of Mt. Bukowa n. Przedborz, Ps Qrp plought cl., 10.73: Mt. \& RO, LOD 20234, 20317, 20368, 20405 \& 20409: - DE 73 f.r. Skrzydlów n. Kłomnice, Qrp Ps Ldp Bpp, 06.79 \& 04.80: Mt. LOD 20222 \& 20785-86: - DE 76 fr. Antoninów Fl Dąbrowa Zielona, Ps, 08.78 : ML. LOD 20227; - DE $77 \mathrm{cnv}$. of Raczkowice, 11.74: ML. LOD 20443: - DE 86 fr. Smykòw n. Swiçta Anna, Ps Qrp Bpp Cb, 05.79: ML, LOD 20784; - EE 00 Slugocice FI Smardzewice, Qrp Aa Ps, 05.74: RO, LOD 20322; - EE 22 Res, Bialaczów n. Opoczno, (bo Bpp Qrp Ps, C8.82: ML, LOD 20769; - EE 40 Wygwizdów, Ps cl., 10.73: RO \& ML, LOD 20413; - EE 41 Lipa n. Cieklinisko, Fs Aa, 04.74: RO, LOD 20226. Wy2yna Kielecko-Sandomierska EE 48 Zębiec n. Ilza, Ps Qrp, 06.79: Mt, LOD 20423: - EE 78 Mi. Cheimowa, Fs $A a, 07.75$ Mt. LOD 20444. Wyżyna Lubelska - FE 37 Zemborzyoe n. Lublin, Ps, 1065: BS, L.BL Pogórze Zachodniobeskidzkic - EF 81 Crastaw 12. Dobczyce 09.10. K. Rouppert. KRAM 878. Beskidy Zachodnie - DG 17 Zawoja - Widly ca $600 \mathrm{~m}$ alt. mixed forest. 09.64: WW, KRAM 15814. Tatry - DG 59 Koscieliska Dale (Dolina K.) mouth of the Smytna Dale, Pa, 10.79: Mt LOD 20306; - DG 59 Kużnice, Pa, 08.58: AS, WA 011962; - EG 50 Rusinowa Glade (R, Polana), Pa, 08.71: S Frejlak, WA 019641. Bieszczady Zachodnic - 
FG 46 Jabłonki n. Cisna, 05.76: Mt, LOD 20228. Pojezierze Mazurskic - FB 57 Tama n. Rajgród, Ps Pa Je, 08,79: RO, LOD 20410. Nizina Polnocnopodlaska - GC 55 BiNP f.s. 369, Ps, 05.78: Mt, LOD 20240.

\subsection{Elaphomyces asperuhis Vitt.}

Distribution in Europe (Fig. 3). Occurring commonly and frequently in $\mathrm{C}, \mathrm{E}$ and $\mathrm{N}$ Europe, also known from northern France (vicinity of Paris), $\mathrm{E}$ Alps, $\mathrm{S}$ and $\mathrm{N}$ slopes of Alps, and the entire Carpathian Mtns. In the Tatras, it is the most frequent species of Elaphomyces, similar to the situation found in Scandinavia. The most northern locality is close to Narvik in Norway, whilst the extreme limits, Kuusamo, in Finland are a little to the south. The highest limits are Strbské Pleso (Tatras) at $1100 \mathrm{~m}$ alt., and Pfronten n. Breitenberg in the Bavarian Alps at $1400 \mathrm{~m}$ alt. No data exists

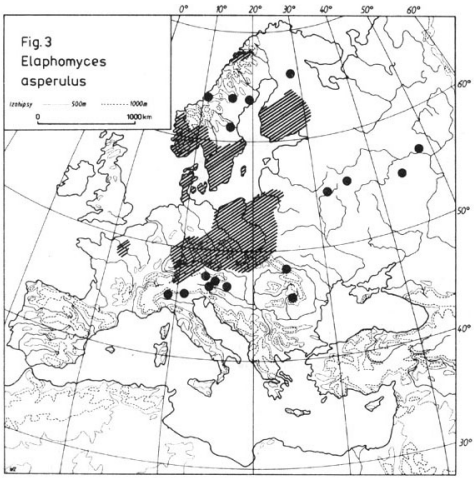




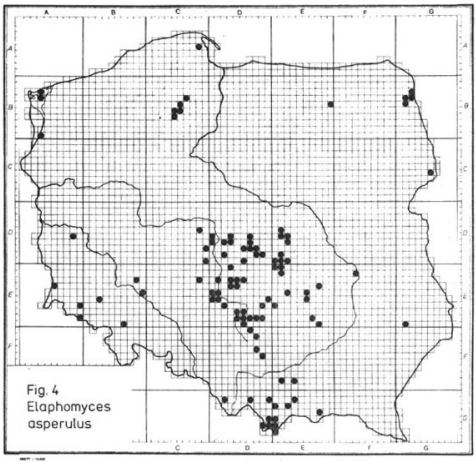

for southern Europe, the Hungarian lowland, most of W Europe, or the Steppe regions of E Europe.

Hypothetical distribution. Prefers regions with a temperate, cool or continental climate. Probably occurs throughout the range of Pinus sylvestris and Picea abies. The highest localities occur in montainous regions; in E Europe it represents the most common species of Elaphomyces. Absent from areas with a Mediterranean or Atlantic climate, and in the Steppe zone.

Habitat. Ectotrophic mycorrhizal associations formed primarily with conifers: Pinus sylvestris, Picea abies, and Abies alba, sometimes probably also with deciduous trees, such as Quercus, Betula, Fagus and Carpinus. Often extreme habitats are attained: over $1000 \mathrm{~m}$ alt. in mountainous regions, and within poor habitats for pine forests in lowlands where $E$. granulatus is a rather rare species; $\mathrm{pH} 4-4.5$. It is the species of Elaphomyces 
most strongly associated with poor, acid habitats of pine and fir forests, and mixed oak-pine forests. It occurs together with E. granulatus, more rarely with $E$. muricatus, Hydnobolites cerebriformis, and the sclerotia of Cenococcum geophilum. Sometimes, but more rarely than in the case of E. gramulatus and $E$. muricatus, the fruitbodies are attacked by the stromata of Cordyceps capitata and C. ophioglossoides.

Distribution in Poland (Fig. 4). Currently known from 127 localities distributed throughout Poland. A larger concentration appears in the Bory Tucholskie (Tuchola Coniferous Forests), Puszcza Białowieska (Bialowicża Primaeval Forest). regions of older glaciations in the lowlands, as well as in the Beskid and Tatra Mtns. No data are available from regions of the last glaciation and little is known from the Silesian Lowland i.e. regions of mostly loams and loesses.

Localities in Poland. Pobrzeze Baftyckie - AB 23 WoNP Wickowo, PQ FQ LF Ps, 06 \& 09.74: RO. LOD 20291 \& 20471-72: - AB 33 Lubin - Traciągowo, Fs, 09.74: RO, LOD 20473; - AB 93 Puszcza Bukowa Res. Bukowe Zdroje, Fs, 06.74: M1, LOD 20254 \& 20470; CA 58 Puszeza Daržlubska n. Reda, Fs, 09.69: MŁ LOD 20292. Pojezicrze Poludniowo. pomorskie - CB 36 Bory Tucholskie Res. Odry. Ps, 07 \& 09.74: ML, LOD 20474-81: - CB 45 Peatbog (Torfowisko) Kwiekı F1 Czersk, Bpn, 08.74: Mt, LOD 20298; - CB 54 Spicrewnik, Ps, 07.73: Mt, L.OD 20466; - CB 55 Zukowo, Ps cl. 07.73: Mt, LOD 20465: - CB 64 Raciąski Mlyn, Ps Bpp cl., 07.73: ML, LOD 20264, 20297 \& 20464, Wzniesienis Zielono. gorskic - AD 58 Zielona Góra, 06.75: W Makowski, LOD 20467. Nizina Poludniowowielkopolska - CD 48 Wrąca n Turck, Ps. 10.78: Mt, LOD 20204; - CD 79 Sokotów n. Kożminek, Fs Bpp Cb, 10.78; ML LOD 20199, - CD 99 Wojkowo F1 Brąszewice, $F_{s}$. Aa Ps, 07.75: RO, LOD 20385; - CE 28 Res. Ryśn. Lututów. Fs Cb Aa Pa, 07 \& 10.79 KM, Mt \& RO, LOD 20248.49, 20265-66, 20274, 20374 \& 20489; - DD 50 Evinów n. Unicjów. $P_{s} C b$ Qrp. 04.75 \& 10.78: ML, LOD 20200 \& 20386; - DD 53 Gostków n. Poddębice, Ps Pa Qrp. 04.75: Mt, LOD 20349; - DD 60 Linne n. Dobra, Ps Qrp Pa, 04.75: MŁ, LOD 20262 \& 20359; - DD 62 Poduębice - Uniejón, Ps Qrp Pa, 04.75: Mt, LOD 20679; - DD 63 env. of Res. Jodly Oleśnickic, Ps, 07,82: KM, LOD 20723.25; - DD 75 kódż Park Ludowy, Ps Bpp Qrp. Pt Ch, 10.79. 06 \& 07.82. KM \& MK. LOD 20398 \& 20674-77: - DD 76 Lódź Park Mickiewicza, Qrp Bpp Ps Rp, 10,79: KM, LOD 20399: - DD 82 f.t. Wojslawice n. Szadek, Psi, 10.79 \& 81: MK, ML \& KM, LOD 20261, 20380-81, 20393 \& 20720-22: - DD 95 Slądkowice .n. Kolumna, Ps Qrp BPp, 06.79: RO, LOD 20441, - f.r. Puwlikowice, Ps, 06.79: RO, LOD 20484: - DE 03 FI Sedziejowice, Ps, 11.73: Mh, LOD 20387 \& 20392; - DE 21 Konopnica, $P a, 07.77$ : KM, LOD 20288; - DE 23 Bagno n. Zar, Ps, 09.78: RO, LOD 20487; - DE 24 Kluki, Ps Bpp Pa Qrp cl., 08.78: Mt, LOD 20482; - DE 24 env, of Kurnos n. Beichatow, Ps cl., 06.80: RO. LOD 20205: - DE 25 Nowy Swiat, Ps cl, 11.76: Mt, LOD 20289; - DE 33 f.r. Stróża n. Szczerców, Ps, 06.78: RO, LOD 20252; - DE 34 fr. Wola Wydrzyna, Ps Qrp Bpp Cb Pa, 09 \& 10.79: KM, LOD 20272-73, 20305 \& 20373. Nizina Ślaska BE 49 Wroctaw Ogród Botaniczny, 05,1869: Herb. Schroet. WRSL. Nizina Środkowomazowiecka - ED 41 Puszeza Bolimowska f.f. Bolimów, Ps Cb, 05.82: MK, LOD 20684-88, - Ruda Sanice, Ps, 08.82: RO, LOD 20715; - ED 52 f.r. Puszcza Marianiska, Ps Qrp. Cb, 09.79. 05 \& 0682: MK, KM \& E. Wiącek, LOD 20304 \& 20689-714. Wzniesienia Południowomazowieckic - DD 56 f.r. Szcawin FI Grotniki, Ps el., 02.74: J. Jozefowicz, LOD 20290; - DD 66 Lódż Las Lagicwniki, Ps Pa, 03 \& 04.74, 05.77: 
M. Paziuk \& L. Samosiej, LOD 20247, 20357, 20390-91 \& 20823; - DD 77 f.r. Wiączý́ n. Nowosolna, Ps Bpp Fs Aa Qrp Pa, 05-10.75: KM, LOD 20279-86, 20294-95 \& 20382-83; - DD 87 Justynów n. Łodz Ps Fs, 09,77: KM, LOD 20439, Qrp Bpp Ps, 10.79: ML LOD 20299; DD 87 Res. Galków, Cb Fs Orp. 1081: KM. LOD 20678; - DD 88 Będzelin n. Rokiciny Aa Cb. 05,74: ML, LOD 20490; - ED 62 Res. Babsk, Ps Qrp Cb Tc, 08.79, 07 \& 09.80: KM, LOD 20394, 20463 \& 20682-83: - ED 81 Wielka Wola F1 Spala. Ps Qrp. 0881: RO. LOD 20509:ED 90 fr. Cygan n. Tomaszów Mazowiecki, Ps cl, 06.80: Mt, LOD 20195 \& 20681: - ED 91 Res. Spała, Ps Qrp. 06.69 \& 04.76: Mt \& RO, LOD 20351 \& 20468, - f.r. Spala. Cb Qrp. 05.77: MŁ. LOD 20353, - Królowa Wola. Ps Pa, 05.77: Mł. LOD 20360. Pozórze Zachodniosudeckie - AE 35 7gorzelec, M. Sudety Zachodnic - AE 69 env. of Jelenia Gota, M; AE 89 Pot-hole of Snicżka Mt. (Kociot Sniczki), Herb, Rehm KRAM, M, S, WRSL. Wyzyna Woźnicko-Wieluniska - DE 40 f.r. Mierzyce, Ps Qrp Fs, 06.79 \& 07.82: RO \& MK. LOD 20717-19, - Kamionk a FI Mierzyce, Ps Qrp Pa, 04.75: ML LOD 20388-89; - DE 41 f.r. Nižankowice, $\operatorname{Qrp} P s, 09.75$ \& 05.82: KM \& MK, LOD 20268; - DE 50 Załçcze Mate, Ps Pa Aa Qrp, 07.79: Mt, LOD 20276 \& 20485, - Kluski, Qrp Ps, 09.79: KM. LOD 20300, Kaluze (= Randaly), Ps Qrp, 07.79: RO. LOD 20278, - Springs of Revelation |Źródła Objawienia) FI Cisowa, Pa, 07.79; RO, LOD 20270, - DE 51 Giętkowizna n. Parzymiechy, $P_{s}$ $\mathrm{Pa}, 09.76$ : ML LOD 20507; - DE 62 Micdzno n. Klobuck, Pa, 09.79: ML, LOD 20267. Wy fyna Krakowsko-Crestochowska - DE 74 Jaskrow. Fs P $, 10,79:$ M1. LOD 20263: - DE 84 Mirów - Hektary, Qrp Pt, 08.81: RO. LOD 20260, - Res. Zielona Góra, Fs, 09.82: KM. LOD 20726; - DE 95 Res. Sokole Góry n. Olsztyn, Fs Ps Ch, 0678 : ML, LOD 20251; DF 06 Krocyce Rocks (Skalki Kroczyckie), Cb Fs, 09.82: ML \& MK, LOD 20728-29; - DF 17 Sierbowice - Pradla, Ps Bpp Pt, 09.82: KM, LOD 20727; - DF 37 Jaroszowiec Olk uski, Ps ImP, 05.63: WW, KRAM 15817; - DF 48 OjNP Ravine (Wąwóz) Kurytania n. Ojców, PQ. 07.61: WW. KRAM 13106. Wyžyna Przedborska - DE 59 Stara Wies n. Przedbórz. Ps Qrp Aa, 11.73: Mt LOD 20350, 20384, 20395 \& 20680; - DE 68 Res. Dębowiee n. Maluszyn, TC Pa Bpp Qrp. O\& \& 11.70, 05.81: ME \& KM, LOD 20440, 20459-60 \& 20508; - DE 75 Skrzydlow n. Klomnice, F× Qrp, 04.80: LOD 20730; - DE 85 FD Stawki FI Potok Zloty, Aa $P_{t}$ Cb Bpp Qrp Ps, 10.78: RO, LOD 20201-03; - DE 86 f.r. Wielki Las n. Święta Anna, Qrp Cb Fs Pi Ag Tc, 10.79: Mt, L.OD 20375 \& 20442, fr. Okrąglik n. Przyrów, Cb Qrp Pa Bpp, 10.78: RO, LOD 20206: - DE 87 Rés. Borek, Ps Qrp, 05.79: RO, LOD 20277; - DE 88 Gabrielów n. Koniecpol, $P$ s $P a, 04.75$ : Mt. LOD 20462; - EE 00 Shugocice - Bratkow FI Smardzewice, Aa, 05.74: RO. LOD 20358. - Slugocicc, Aa Pa, 05.74: ML LOD 20255; - EE 11 Prymusowa Wola. Pa Qrp, 08.73: Mt, LOD 20356. - env, of Kamien FI Smardzewice, Qrp Cb, 05,74: RO, LOD 20352: - EE 42 Sielpia Wielka. Ps, 11.73: ML. LOD 20293; - EE 60 Żeleżnica n. Przedbórz, Aa, 04.74: RO \& Mt, LOD 20296. W yżyna Kielecko-Sandomierska - EE 37 Osiny - Pomorzany env. of Ilza, Ps, 0679: M1, LOD 20378: - EE 45 Res. Dalcjón n. Blizyn, Aa, 06.79: ML \& KM, LOD 20197-98, - Res. Cisowy Majdów, Ps Aa, 06.79: ML LOD 20303: - EE 55 Res. Świnia Góra, Qrp Ch Aa, 06.79: ML \& KM. LOD 20488: - EE 86 Daleszyce - Cisów n. Wymystów, Aa Fs, 07.77: ML. LOD 20253; - EE 87 Ratków - Hancza. Qrp Ps. 07.77: Mł, LOD 20256, 20269 \& 20731. Wyźyna Lubelska - FE 13 env. of Pulawy. Qrp Ps, 11.53: H. Stasiak, WA 13562. Roztocze - CE 91 Res. Bukowa Göra n. Zwicrzynice, Aa, 09.66 \& 07.68: BS, LBL. Pogórze Zachodniobeskidzkie - EF 81 Czastaw n. Dobczyce, 04.11: K. Rouppert, KRAM 877; - EF 83 Res. Kamienie Brodzińskiego n. Bochnia, PQ, 05.74: WW, KRAM 15046. Beskidy Zächodnie - DG 12 Barania Mt. Aa Pa 05.58: AS, WA 011956: - DG 16 BaNP $1080 \mathrm{~m}$ alt., Pa. 06.61: BG. KRA. Beskidy Zachodnie - DG 19 Rabka, Pea, 11.79: MŁ, LOD 20196; - EG 13 Kámicnica Dale (Dolina Kamienicy), $\mathrm{Pa} A a, 06.76$ : Mt, LOD 20354; - EG 20 Bukowina Obidowska, Pa, 07.67: WW, KRAM 13105; - EG 22 env, of Maniowy n. Nowy Targ $700 \mathrm{~m}$ ait. 08.63: WW, KRAM 13104: - EG 37 Tylice, Ad, 10.60: BG, WA 009581.04.61: BG, KRA, - Tylicz - Krynica, Aa. 07.60 
\& 09.62: BG, KRA. Tatry - DG 49 Kalatówki cil $1200 \mathrm{~m}$ alt. Po, 07.64: WW. KRAM 15818; - DG 58 Chocholowska Dale Molkówka, Pa, 10.79; Mt, LOD 20193, 20257-58 \& 20396; DG 59 Ku Dzurze Dite. Pa. 1079: Mt. LOD 20301, - Dale of Bialy (Dolina Bialego). Pa. 10.79: Mt, LOD 20259 \& 20302, - Lejowa Dale, Pa, 0x.82: ML. LOD 20734, - Strazyska Dale, $P a$ Aa Fs, 10.79: Mt, LOD 20486 - Kosicieliska Dale month of the Smytna Dale I080 $\mathrm{m}$ al. $P a, 1079$ ML. LOD 20271. - Mt. Nosal ca $1200 \mathrm{~m}$ alt. Zakopane - Kuf́nice Pu. 05.73: WW. KRAM 15751: - EG 40 Zahopanc Jaszczurówka, Avi Pa, 08.58: AS. WA 011964. - env. of Murzasichle ca $850 \mathrm{~m}$ alt. Pa, 04.71: L. Plewicki, KRAM 15820, - Zakopane Chłabówka, Pa Aa, 06.57: W. Rudnicka-Jezierska, WA 011966; - EG 50 Olczyska Dale $892 \mathrm{~m}$ alt. Pa, 08.80 \& 82: BS \& ML. LOD 20732-33, - Roztokit Dils, Pa. 06.79: ML, LOD 20194, - Rock over the Lysa Glade (Skalka nad tysa Polana). Pad ox 69: S Frejlak. WA 0171xi, Myślenickie Fells (M. Turnic), 08.35: J. Lilpop, KRAM 886:- EG 60 Pot-hole of the Morskie Oke Lake (Kociol Morskicgo Oka), Po. 07 \& $0 \times 58$ \& 66: S. Frcjlak. WA 014756.42 \& 014805 . - Morskic Oko, Pa, 06.56: AS, WA 011965, - Wosjenica Glade ci $1450 \mathrm{~m}$ alt. Pa. 07.72:

2. Heinrich, KRAM 15819. Pojezierze Litewskie - GB 22 Puszcza Augustowska env, of Pliskie Lake, Ps cl. 08.79: RO. LOD 20379; - GB 31 Res. Maly Borck. Ps Pa, 08.79; RO. LOD 20376, - Res, Starożyn, Pa BrF Pt QrP, 08.79: RO, LOD 20250 \& 20377; - GB 32 Rygol, $P s$ Pa Bpr, 08,79. RO, LOD 20397; - GB 41 env, of Res Kozi Rynek FI Balinka, Pa, 06.72: Mt, LOD 20469 Pojezierze Mazurskie - EB 49 Bobrowko n. Mikolajki, Aps Ps Pt Ch, 08.81: RO, LOD 20736. Nizina Pölnocnopodlaska - GC 55 BiNP, TC Pa, 05.78 \& 10.81: ML IOD 20275 \& 20735

\subsection{Elaphomyces muricatus $\mathrm{Fr}$.}

Distribution in Europe (Fig. 5). Common throughout C Europe, southern Scandinavia, and the British Isles. A lowland-upland species, also of low mountain sites. Many localities extend to the southern slopes of the Alps and to the Lombardy heights, but fewer localities are known for the northern slopes of the Alps and no information exists for the E Carpathian Mins. Remarkably the species occurs on the Hungarian Lowland and nearby Kiev. Differentiated into distinct ecological forms, also differing with their geographical distribution. The full range of -forms is limited to $\mathrm{C}$ Europe and southern Scandinavia. Further north, it is found only as f. muricatus and f. reticulatus, with localities around the Gulf of Bothnia, and the northernmost locality at Kittila, $100 \mathrm{~km}$ beyond the arctic circle (almost $68 \mathrm{~N}$ ). In the extreme southern localities, only f. variegatus and f. fagicolus in the Fagus range are represented.

Hypothetical distribution. A typical deciduous forest species of the nemoral zone of maritime and submaritime climate. Also, in a more continental climate, approaching the forest-steppe border.

Habitat. Ectotrophic mycorrhizal associations formed with several tree species: often found in mixed forests, occasionally in coniferous forests. Ecological forms with morphologically differentiated fruitbodies are associated with specific trees, such as Fagus syltatica, Carpinus betulus, Quercus rohur, etc. Occurring in lowlands and low mountain sites, in compact, fertile 


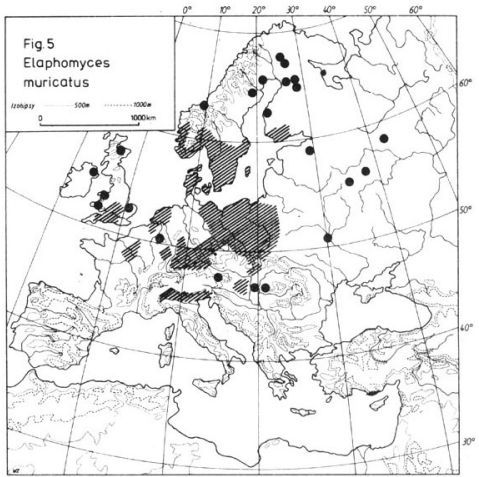

soils, with a $\mathrm{pH} 4.5-5$. Frequently found together with $E$. granulatus, more rarely with $E$. asperulus, and the sclerotia of Cenococcum geophilum. The fruitbodies are sometimes attacked by Cordyceps capitata and C. ophioglossoides. In the vicinity, Hydnotrya tulasnei, Genea hispidula, and some species of Tuber can often be found.

Distribution in Poland (Fig. 6). Frequent in lowlands but disappearing in low mountain sites. Until now considered rare in the mycoflora, and known only from individual localities. According to the author's investigations, it appeared to be the most common species of the hypogeous Ascomycetes, and was found in 140 localities.

Localities in Poland. Pobrzeże Battyckie - AB 23 WoNP FD Miedzyzdroje, Fs Qrp FQ, 10.62: M. Lisiewska, POZ, - Wiselka, Qrp Cb FQ. 06.77: ML, LOD 20155; - AB 33 


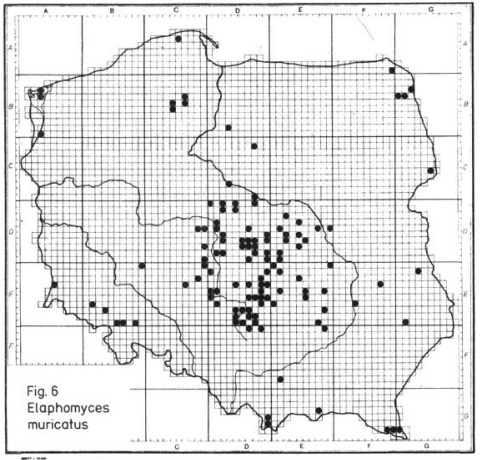

Lubin - Traciagowo, F5, 06.74: M L LOD 20149; - AB 93 Puszeza Bukowa Res. Bukowe Zdroje, FQ, 10.61: M. Lisiewska, POZ. - Podjuchy, Fs, 06.74: ML, LOD 20054, 20082, 20164 \& 20332; - CA 45 Choczewo, Fs, 07.82; Mt, LOD 20655-56 \& 20656. Pojezierze Poludniow opomorskie - CB 36 Res. Odry, Ps Bpp, 10.73: ML, LOD 20167; - CB 44 Bory Tucholskie Konigort, Cb Qrp Tc, 08.74: ML, LOD 20152; - CB 46 Gotelp, Fs Qrp, 07.74: Mt, LOD 20330 - CB 54 Rytel, Ps $\mathrm{cl}_{7}$ 07.73: Mt, LOD 20429, - env. of Gluche Lake, Bpp Ps, 08.74: Mt, LOD 20153. Pojezierze Chelminsko-Dobrzyńskie - DB 83 Orle FI Ossa n. Rogoźno, Cb TC, 07.82: RO, LOD 20657: - DC 17 Res. Las nad Branica n. Górzno, Cb, 07.82: RO, LOD 20673. Pradolina Torunsko-Eberswaldzka - DC 73 Wloclawek, Fs, 09.79: RO, LOD 20324; - DC 97 Res, Lack, Ps Orp Cb, 07.79: RO, LOD 20436; - DD 07 Zdworsk Fl kack, Ps Qrp, 09.79: RO, LOD 20425. Pojezierze Wielkopolskie - DD 00 Res. Kawęczyriskie Brzęki n. Lubotyñ, Cb Ps, 10.78: ML, LOD 20671; - DD 02 f.t. Rogoźno, Ps Qrp, 09,79: RO, LOD 20094, 20493 \& 20672; - DD 04 Siemianów n. Lubień Kujawski, Qru PaQ, 09.79: RO, LOD 20433. Nizina Południowowielkopolska - CD 48 Patnów. Te Po Aps. 10.78: ML LOD 20159; - CD 49 Malaszyna, Qrp Ps PoQ, 10.78: ML, LOD 20160; CD 99 Jasionna n. Blaszki, Qrp Cb Ps, 08.79: KM, LOD 20116; - CD 79 Sokolów n. 
Kożminck. Ul Pa RP Qrp Fs, 10.78: RO \& Mt, LOD 20634-35: - CE 09 Kliczków Forests (Lasy Khezkowskie) n. Jarozlaj \& Sundaly. P、 Qrr, 08.79 : KM, LOD 20006 \& 20111: - CE 28 Res Rvin n Lutulow. Fi A I Pa. 0779 \& 09.80; ML \& RO. LOD 20081, 20083. 20336 \& 20516: - CE 36 Biadaszki n. Opatów, Qrp F: Ch, 01.81: RO, LOD 20172, - Ustronic, Qrp, 09.80: Mt. LOD 20518; - DD 12 f.r. Kobylatke F1 Kolo, 07,79: RO, LOD 20004, 20075 \& 20085; DD 14 Ostrowy n. Kutno, Qrp Ch, 06.79: ML, LOD 20090 \& 20106; - DD 31 Dąbie, Qrp Ch 10.78: ML LOD 20426: - f.r. Wielenin, Qrp Ch, 1078: M1, LOD 20158 \& 20670: - DD 52 env: of Poddębice. Qrp. 04.75: Mt. LOD 20052; - DD 62 Zakrzew n. Poddębice. Qrp Ps. 0580: KM. LOD 20438: - DD 65 f.r. Krogulec FI Grolniki. Bpp Ps Qrp, 07.74: J. Wesclowski, LOD 20028: - DD 75 tódź Park Ludowy, Qrp Bpp Ps Ch Pe. 05.77. 10.78 \& 0682: Mı, KM \& MK, LOD 20042 \& 20567-75: - DD 76 Lódż Park in. A. Mickicwicza, Qrp Ps Bpp Aps, 10.79: KM. LOD 20025: - DD 81 Rossossyca, Ps, 04.75: Mt, LOD 20119 \& 20347: - DD 82 f.r. Wojslawice n. Szadck, Qrp Ps Aa Cb Pa, 10.79, 07 \& 10.81: MK, KM M1 \& RO. LOD $20011-12$ 20023, 20096, 20114, 20150-51 \& 20636-38; - DE 10 Res. Nowa Wies, Fs, 10.80: ML LOD 20519: - DE 34 fr. Wola Wydrzyna n. Szczerców, Ch Qrp Bpp Aa Ps 06.78, 09 \& 10.79, 08.80: KM, ML \& RO, LOD 20026, 20084, 20100, 20105, 20166 \& 20642; DE 36 f.r. Pytowice FI Kamiensk, Qrp. 08.78: ML, LOD 20087. Obnizenic Milicko* -Glogow skie - BE 99 Niezgoda n. Zmigród, Qrp, 06.82: E. Wiącek. LOD 20666. Nizina Środk owomazowiecka - DD 46 f.r. Bielawy - Mroga. Qrp. 10.58: W. Rudnicka-Jezicrska. WA 012650: - ED 22 Teresin n. Szymanów, Cb Qrp. 0781: RO, LOD 20514: - ED 34 Podkowa Leśna, Qrp. 10.70: ML. LOD 20059:- ED 41 Pusscza Bolimowska f.r. Bolimów, Qrp Ps, 05.82: MK \& KM. LOD 20578 \& 20588; - ED 47 Chojnôw, Qrp Ps, 10.58: A. Wosinska, WA 008845; - ED $49 \mathrm{fr}$. Calowanie, Ag, 07.63: K. Nowak, WA 016022; - ED 52 Puszcz: Marianska, Ch Ps, 06.82: MK, KM \& E. Wiacek, LOD 20579-87, 20590-91, 20595-96 \& 21118: - ED'52 Res. Modrzewina n. Mala Wies, $C b, 06,79:$ Mt \& KM, LOD 20015: - EE 09 Res. Zagozdzon n. Kozienice, Qrp. 10.72: BS, LUB. Wzniesienia Poludniowomazowieckic DD 59 Res. Bukowice n. Bobrowa, Fs Qrp, 05.74: RO. LOD 20060; - DD 66 Lodz Las tagiewaiki, Ps Orp Fs Pa, 03-10.74 \& 07.77: KM, M. Paziuk \& Z. Lawnicka, LOD 20027, 20029-30, 20032-40, 20062, 20072, 20108 \& 20576-77, - todż Rogi, Qrp Pa Cb, 12.78: RO, LOD 20513; - DD 67 f.r. Janinów n. Brzeziny, Fs Qrp, 04-11.74, 03.75 \& 10.78 : Mt, RO \& E Najmanowic, LOD 20049, 20069, 20089, 20102, 20157, 20325, 20339, 20345 \& 20630-31: - DD 69 Rogów, Qrp Aa, 11.74 : ML. LOD 20068, - Res. Zimna Woda n. Rogów. Qrp Ps Cb. 05.74: RO. LOD 20328; - DD 77 f.r. Wiacayn n. Nowosolna. Fs Pa Aa Ps Cb, 07.74, 05-11.75 \& 11.79 KM. ML. RO \& Z. Lawnicka, LOD 20103, 20123, 20127, 20137-39, 20141-48, 20343 \& 20624-28: - DD 87 Res. Galków, Cb Qrp Fs, 10.81: MK, LOD 20629, - Justynów n. Lơdz, Crp Ps Bpp Cb, 08.80; A Lawrynowic,, LOD 20173, - Janowka n. Justynow, Fs Qrp, 08.82: L Samosiej. LOD 20632: - DD 89 Wegrzynowice n. Brzeziny, Qrp, 0972 : Mt, LOD 20047: DD 96 Res. Molenda, Qrp Pa, 08.74: Mt, LOD 20013, 20121 \& 20619: - DD 99 f.t. Kruszeniec, $C b, 05.74$ : ML LOD 20044, 20058 \& 20136; - DE 19 Lubiasðów - Markowice Mokre. Ch PaQ, 08,72; RO, LOD 20348, - fr. Lubiaszów, Ps Qrp TC, 05.80: RO \& G. Drzeri, LOD 20041 \& 20515; - DE 28 f.r. Kulen n. Leczno, Qrp. 10.72: ML. LOD 20048, 20163, 20335 \& 20434: - DE 38 f.r. Lubien, Ps, 09.72: MŁ. LOD 20430; - ED 54 Skuły Wschód, Cb . Ag, 09.81: RO. LOD 20510 \& 20597; - ED 62 Res. Babsk. Co Qrp. 07 \& 08.79 , 04-09.80: KM, LOD 20005, 20016-17, 20022, 20078-79, 20109-10, 20112, 20517 \& 70598-607; - ED 74 Res. Trebaczew, Qrp Cb Ldp Ps PaQ, 08.68,04-08.69, 08 \& 11.70, 04.72 \& 06.79 : ML. \& KM, LOD 20009, 20019, 20044, 20056, 20120, 20122, 20124-26, 20128-29, 20131-32, 20435 \& 20608-13:ED 81 Wielka Wola FI Spata, Qrn Bpp Ps. OR.81: RO. LOD 20620 \& 20622; - ED 91 Res. Spala, Qrp Fs Ps Bpp Pl. 10.68, 08 \& 11-12,69, 04.76: Mt. \& RO, LOD 20053, 20133-35 \& 20338, - f.r. Spala Cb Bpp Qrp Ps, 11.73, 05.77 \& 10.81: ML, RO, MK \& J. Guzewski, LOD 
20055 \& 20614-16, - Res. Konewka n. Spata, Qrp Ps. 05.74 \& 08.82: ML \& RO, LOD 20130, $20337 \&$ 20617-18; - EE 00 Borki n. Spala, Cb Ps, 11.73 \& 06.74: ML, LOD 20046 \& 20115. Przedgórze Sudeckie - BE 98 Res. Muszkowicki Las Bukowy, Fs, 06.82: RO, LOD 2092223. Sudety Zachodnie - BE 61 Sokole Mtns n. Trzcirisko, ca $500 \mathrm{~m}$ alt., Pa, 06.62: WW, KRAM 15816, Sudety Srodkowe - BE 73 Szczawno Zdeoj, Cb Qrp Fs, 04.82: KM, LOD 20658-59; - BE 95 Sowie Mtns, 08.24: M; - BE 96 Bielawa, Qrp Tc Aps, 08.81: KM, LOD 20334. Wyżyna Woznicko-Wieluniska - DE 40 Mierzyce, Ps Qrp Pa, 06,79, 05 \& 0708.82: RO \& MK, LOD 20003, 20437 \& 20639-41; - DE 50 Res. Stawiska n. Parzymiochy, Qrp, 07.72: J. Herénniak, LOD 20050, - Res. Bukowa Góra, Fs, 04.74: Mt, LOD 20331; - f.r. Nizankowice, PS Qrp, 09.79: ML \& KM, LOD 20024. Wyżyna Krakowsko-Czętochowska - DE 74 Jaskrów, Qrp FS BPp, 10.79: ML, LOD 20095; - DE 84 Mirow Hektary, Qrp Pt Fs, 08.81: RO, LOD 20326, - Res. Zielona Góra, Fs Ps, 08.81: MŁ. LOD 20333; - DE 94 Res. Sokole Góry n. Olsatyn, Fs, 06.78: M1, LOD 20088; - DE 95 Polok Zloty, Fs, 09.82: ML. MK, W. Malinski, LOD 20643-46; - DE 06 Krocryce Rocks (Skałki Kroczyckic), Fs, 09.82: ML, MK \& KKM, LOD 20647-48; - DF 08 Ohadza FI Szczekociny. Qrp Ps, 09.82: Mt、MK \& KM, LOD 20652. Wyžyna Przedborska - DE 19 Res. Gaik, Qrf. 04.78: ML \& RO: LOD 20086 \& 20171; - DE 39 Diabla Góra n. Opoczno, Qrp Ps, 10.73: ML. \& RO, LOD 20065, 20170 \& 20623; - DE 48 Res. Bąkowa Góra, Qrp Fs, 1079: RO \& Mt. LOD 20098 \& 20426; - DE 56 Res, Jasien, $P s$ Pa BPp, 06.75 \& 05.83: M , LOD 20107: DE 57 Kajetanów FI Kobiele Wielkie, Qrp Ps Bpp Aa, 09.78: RO, LOD 20007 \& 20432; - DE 58 Res. Góra Chelmo, Qrp Cb Ps, 0878 : Mt, LOD 20010, 20020, 20156 \& 20341; - DE 59 Res. Bukowa Góra, $F 5,10.73$ : Mt, LOD 20118, - Stara Wies, Qrp Ps, 11.73: Mt, LOD 20057: - DE 68 Res. Dębowiec, Qrp Cb Bpp. 06.79: Mt \& KM, LOD 20001 \& 20018: - DE 75 Skrzydlow, Qrp Bpp Qru, 06.79: ML, LOD 20014 \& 20092: - DE 76 Antoniow FI Dabrowa Ziclona, Qrp, 08.78: ML \& RO, LOD 20511; - DE 86 f.r. Wielki Las n. Swięta Anna, Cb Fs Bpp, 05.73 \& 10.79: Mt \& RO, LOD 20008 \& 20329, - f.r. Knicja. Ag, 10.79: M1, LOD 20077: - DE 87 Res. Borek, Qrp. 04.75: Mt, LOD 20066 \& 20091; - DE 97 f.r. Wygicłzów n. Lelów, Fs, 09.82: ML LOD 20653, - Zagórze n. Sokolniki, Fs, 09.82: RO. LOD 20654: - DF 08 fr. Gaszcze FI Szczekociny, Cb Pt Bpp Qrp, 09.82: KM, ML \& Z. Wnuk, LOD 20649-51; EE 00 Slugocice FI Smardzewice, Cb Qrp Aa Ps, 05.74: ML \& RD. LOD 20064, 20117, 20327, 20340. 20346 \& 20633: - EE 11 Prymusowa Wola, Qrp Ps Pa, 08.73: Mt, LOD 20104 \& 20621, - Januszewice, PaQ, 08.74: RO, LOD 20113; - EE 41 Lipa n. Ciek lińsko, Fs Aa, 04.74: M $\downarrow$ LOD 20169; - EE 60 Res. Zabrody n. Olesno, $P a, 11.73:$ Mt, LOD 20063. W y y na Kielecko-Sandomierska - EE 24 Przysucha, Qrp Fs, 05.74: RO. LOD 20l61-62: - EE 45 Res. Skarżysko, Fs Fe Ag. 06.79: ML LOD 20342: - EE 48 Zebice n. Ijża, Ps Qrp Cb. 06.79 MŁ \& KM. LOD 20071: - EE 55 Res, Swinia Góra, Aa Qrp, 06.79: ML, LOD 20045 \& 20431: - EE 78 Res. Göra Cheimowa, 04.10: M. Raciborski, KRAM 885, - Fs, 07.75: Mt, LOD 20140; - EE 97 Raków - Jamno, Qrp, 07.77: MŁ, LOD 20073, - Raków - Hanicza, Qrp Ps Po, 07.77: Mt, LOD 20074, 20323 \& 20663; - EF 08 env. of Staszów, Orp Ps, 06.77: ML, LOD 20165, Wyżyna Lubelska - FE 37 Zemborzyce n Lublin, Qrp Cb, 08.67: BS, LBL: - FE 63 Stary Rachów, Qrp, 09 \& 10.65: BS, LBL - Grabówka n. Annopol. Qrp, 10.65: BS, LBL. Roztoçe - GE 91 Res. Bukowa Góra n. Zwierzynicc. Fs Ag. 10.66, 08.67 \& 08.68: BS, LBL. Pogórze Zachodniobeskidzkic - EF 81 Czasław n. Dobczyce, Ps Bpp. 09.10 \& 04.11: K. Rouppert, KRAM 884 \& 12882 . Beskidy Zachodnie - EG 37 Tylicz - Krynica, Aa, 10.62: BG, KRA. Tatry - DG 49 Mt. Gubalówka, Pa, 08.80: AS, LOD 20665: - DG 59 Dale of Bialy (Dolina Bialego), Pa Aa Fs, 10,79: Mh, LOD 20021. Bieszczady Zachodnie - FG 68 Wetlina, Fs, 09.58: BG, KRA: - FG 69 Caryniska Mt. Pasture (Polonina C.), Fs, 05.76: ME; LOD 20660; - CG 60 Sreroki Peak (S. Wierch), Fs Pa, 08.76: Mt. LOD 20661-62. Pojezierze Litewskie - FA 99 Rybalnia, Qrp Pa Bpp. 08.79: RO. LOD 20080 \& 20154; - 
GB 22 env. of Rygol, Ps Bpp, 08.79: RO. LOD 20002; - GB 30 Czarny Brod FI Plaska, $P$ s Bpp, 06.72: Mـ LOD 20067: - GB 31 Res. Staroz̉yn, Cb Qrp TC, 08.79: RO, LOD 20076. Nizina Pólnocnopodlaska - GC 55 BiNP, $\mathrm{Cb}$ Tc, 05 \& 10.81: KM \& M, LOD 20512 \& 20667-69. Polesie Woly hiskie - GE 13 Res. Bachus fi. Cheim, PaQ, 08.72: BS, LBL.

\subsection{Elaphomyces papillatus Vitt.}

Distribution in Europe (Fig. 7). On the basis of herbarium material, only two localities are definitely confirmed: one in Poland and the other in Hungary (Libickozma Komitat Somogy). Further localities have been quoted by Szemere (1965). In the Paris herbarium (PC), there are two samples without indicated localities, but annotated "Ex Musteri Vittadini celebrissimis Tul. dedit". Probably they originated from N Italy since the region is

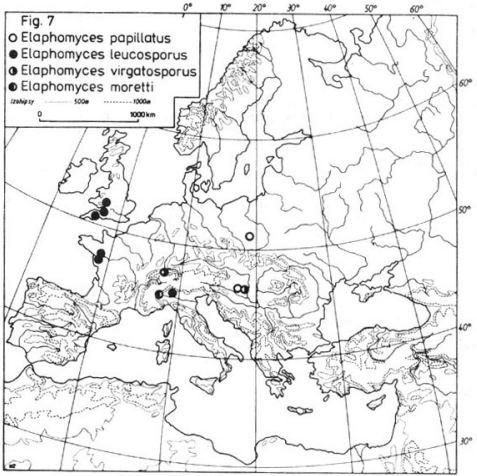


quoted in Vittadini (1831), whilst in Tulasne \& Tulasne (1851) one read: "Vidimus siccum e Lombardia benevole ab ill. Vittadinio nobisc. comm." According to this work the species occurs in oak and chestnut forests nearby Milan and Ticino. Hesse (1894) cites it from Kirchditmol ( $\mathrm{n}$. Kassel, prov. Hessen-Nassau). The Polish locality is the most northerly in Europe.

Hypothetical distribution. Probably quite widely distributed in the mild climatic zone of $\mathrm{C}$ and $\mathrm{S}$ Europe, but rare everywhere.

Habitat. In deciduous forests, under Quercus robur, Q. cerris, Castanea sativa, Betula pendula, Carpinus betulus, and Fagus sylvatica, in soil with a light litter cover. Until now, found only in localities containing other hypogeous species, possibly implying the need for optimal hypogeous conditions. Fruitbodies few in number when found.

Distribution in Poland. (Fig. 8). Appearing in the richest habitat,

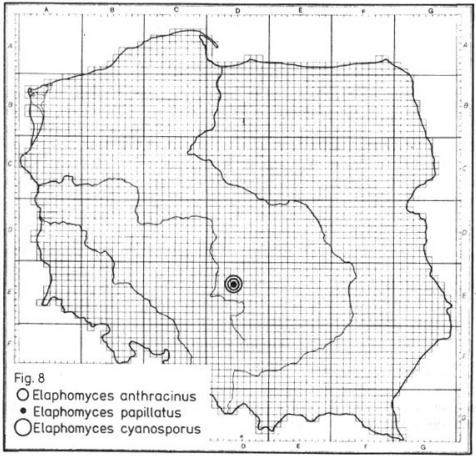


with respect to other hypogeous fungi: together with 11 other species of hypogeous Ascomycetes.

Localities in Poland. Nizina Poludniowowielkopolska - DE 34 f.r. Wola Wydrzyna n. Szczerców, Bpp Cb Qrp, 09.79: RO, LOD 21107.

\subsection{Elaphomyces atropurpureus Vitt.}

Distribution in Europe. The species not yet carted because of the lack of data on its localities in the herbarium materials. In the Paris herbarium (PC) there are some specimens from the Tulasne collection but without data. Probably the specimens came from ltaly since in Tulas ne \& Tulasne (1851), p. 102, one read: "In nemoribus prope Roncaro agri Mediolanensis ed Torre d'Isola Ticinensis", and then: "Vidimus specim. exsiccata". Ceruti (1960), citing Quelet, reports this species also from the French Jura.

Habitat. Oak and chestnut forests; also pine forests on the lime subsoil. Distribution in Poland. Not yet discovered.

\subsection{Elaphomyces leucosporus Vitt.}

Distribution in Europe (Fig. 7): Following the accessible herbarium documentation the appearance stated only in environs of Milan (Italy) and in France along the lower Loire river. Hawker (1954) quotes the species from England citing the original collection of Broome (Chudleigh. Devonshire) and her own collection: Westridge \& Wotton-under-Edge (Gloucestershire) and Cleeve \& Wraxall (Somersetshire); Schwärzel (1958) mentions environs of Basle (Switzerland).

Hypothetical distribution. Warmer and wet zone of temperate climate in W Europe and northern Italy.

Habitat. Under various species of forest trees: Castanea sativa, Fagus syluatica, Fraximus excelsior, and Quercus; originally described species collected under $Q$. ilex. Fruitbodies formed in the humus layer.

Distribution in Poland. Not yet discovered.

\subsection{Elaphomyces maculatus Vitt.}

Distribution in Europe (Fig. 9). Appearing not too often in C Europe, not crossing Alps in the south. Very rare in Scandinavia; the northernmost locality in environs of Uppsala (Sweden). A larger density of localities is known in the Balaton region (Hungary) and in environs of Paris. 


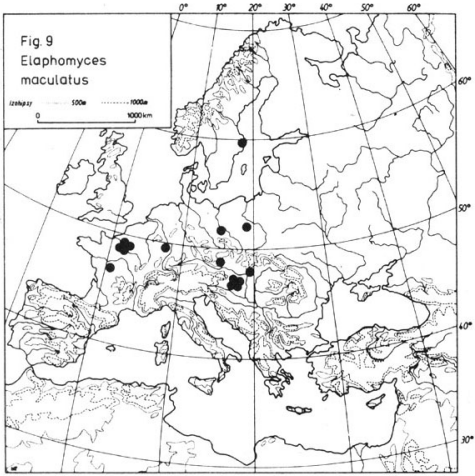

The locality in Poland is one of the three easternmost localities. A typical lowland species, no locality exceeds $500 \mathrm{~m}$ alt.

Hypothetical distribution. The lowland part of C, W \& SE Europe and S Scandinavia, in the deciduous forest zone.

Habitat. Under Quercus, Fagus sylvatica, Carpinus betulus, Alnus glutinosa and Corylus avellana, usually quite deep under the soil surface, in humus of the muil-type.

Distribution in Poland (Fig, 10). Niziny Sasko-Łużyckie - BD 90 Piotrowicen. Przemków, 07.1988: Herb. Schroet. WRSL isub nom. E. anthracinus). Nizina Srodkow oma. zowiecka - DD 46 f.r. Bielawy - Mroga n. Glowno, Cb Qrp, 10.78: ML, LOD 21106. 


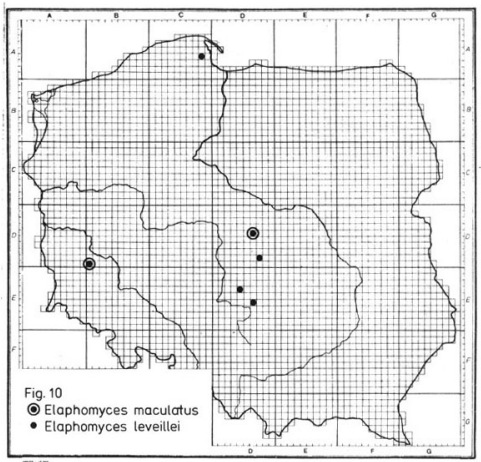

5.8. Elaphomyces leveillei Tul

Distribution in Europe (Fig. 11). Often in N Europe, rare and scattered in C \& W Europe. Appearing in lowlands and low mountain sites. In the north it considerably exceeds the arctic circle; collected nearby Narvik (Norway) and Gallivare (Sweden). When in continental climate, the range is more restricted to the south. The southernmost localities occur in northern Italy and $\mathrm{S}$ slopes of the Alps.

Hypothetical distribution. Entire Europe excluding the S and SE parts, mainly in the boreal zone of deciduous forests.

Habitat. Under Fagus syluatica, Picea abies, Abies alba, and Pinus sylvestris, in acidic, penetrable soils, often in mixed woods including Quercus, Betula, and Castanea sativa. Fruitbodies formed not deep under mossy and lichen-grown soil surfaces. The habitats of the specimens collected by the 


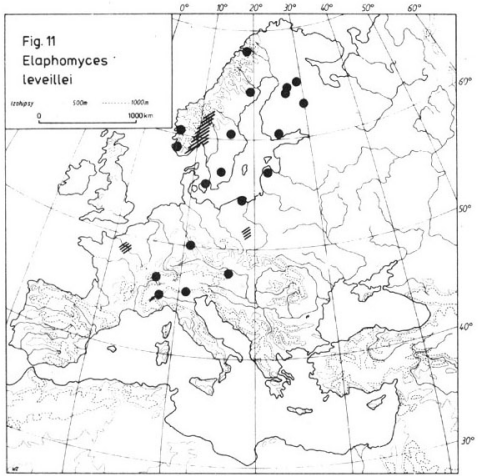

author in Poland and at Gallivare in the far north were quite similar: the undergrowth included the species of Ericaceae: Calluna vulgaris, Vaccinium myrtillus and V. vitis-idaea; in Sweden also Erica sp. and Linnea borealis.

Distribution in Poland (Fig, 10). Pojezierze Wschodniopomorskic - CA 68 Puszcza Darżlubska n. Reda, Fs, 09.69: Mh LOD 21105. Nizina Poludniowowielkopolska - DE 34 f.r. Wola Wydrzyna n. Szczercow, Bpp Qrp Ps, 10.79: KM, LOD 21102. Wzniesienia Poludniowomazowieckie - DD 87 Justynów n. Lodź, Aa Pa, 10.79: Mt, LOD 21101. Wyzyna Przedborska - DE 56 Res. Jasien, Ps Pa Bpp, 05 \& 07.75: M , LOD 21103-04.

5.9. Elaphomyces anthacinus Vitt.

Distribution in Europe (Fig. 12). Not too often in C \& W Europe, Scandinavia and $\mathrm{S}$ from the Alps and Carpathians. Oecurring in lowlands 


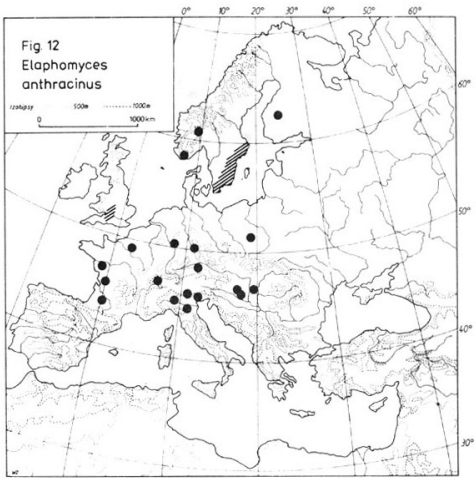

and low tectonic forelands. A considerable density of localities in southern Sweden; in the north not exceeding the arctic circle: the extreme stand at Konnovesi (Finland).

Hy pothetical distribution. Entire Europe except for the Mediterranean $\&$ cold zones and high mountains.

Habitat. Mycorrhiza formed with several deciduous tree species: Quercus, Castanea sativa, Corylus avellana, Fayus syltatica, and coniferous trec species: Abies and Pinus. Fruitbodies formed in the humus layer. In Poland gathered under Carpinus betulus in a sitc poor in herbaceous plants.

Distribution in Poland (Fig. 8). Nizina Poludniowowielkopolska DE 34 f.r. Wola Wydrzyna n. Szczerców, Cb TC, 08.80: RO, LOD 21116. 
5.10. Elaphomyces septatus Vitt.

Distribution in Europe. Not recorded in available herbarium material. Originally described from Italy in Vittadini (1831), p. 68: "In quercetis mediolanensibus secus flumen Lambro". Also reported from Hungary (Szemere 1965) and Denmark (Lange 1956). Hesse (1894) states its absence in Germany.

Habitat. Gathered under oaks, beeches, and hornbeams.

\subsection{Elaphomyces tirgatosporus Holl}

Distribution in Europe (Fig. 7). On the basis of herbarium material, the occurrence ascertained at Szekszard (Komitat Tolna. Hungary). from where the species had been described by Hollós (1908). It was there collected also in environs of Basle (Switzerland) by $\mathrm{K}$ napp in 1921 (M).

Hypothetical distribution. Europe, temperate climate zone of deciduous forests.

Habitat. Fruitbodies gathered under Fagus syleatica and Carpinus betulus.

Distribution in Poland. Not yet discovered.

\subsection{Elaphomyces marptiii Vitt.}

Distribution in Europe (Fig. 7). Only one locality nearby Turin (northern Italy) is recorded in available herbarium metarial. In the original description of the species by Vittadini (1831), p. 71, one read: "In sylva quercina mediolanensi secus flumen Lambro".

Hypothetical distribution. Southern Europe.

Habitat. Oak and chestnut forests.

Distribution in Poland. Not discovered.

\subsection{Elaphomyces aculeatus Vitt}

Distribution in Europe (Fig. 13). Widely distributed. The material from Denmark, FRG, France, Hungary, and Italy has been analysed. Also recorded by Hawker (1954) from England. The most northerly finding was in the vicinity of Stockholm by Kers (1979), the most southerly ones nearby Milan and in the Adige valley in the Alps. Numerous localities are in the southern part of Hungary.

Hypothetical distribution. Not too often, but probably in a wider range than indicated by the currently known localities. Associated with the deciduous forest zone. Throughout Europe, within the temperate climate 


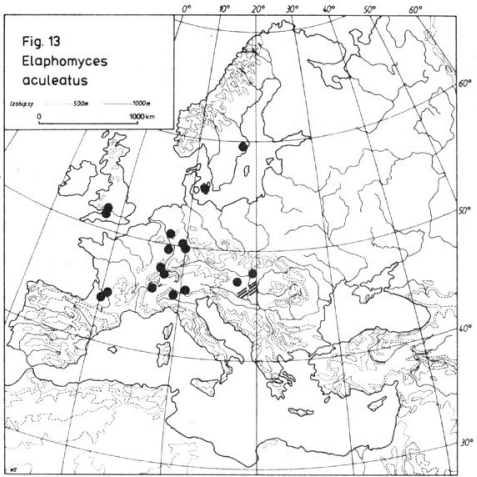

range, especially in its warmer zone, as well as within the zones of maritime and submaritime climate.

Habitat. Under Quercus, Fagus, Fraxinus, and Corylus, often in the lime subsoil. Fruitbodies formed ca $10 \mathrm{~cm}$ under the soil surface, between branched tree rootlets.

Distribution in Poland. Not yet discovered, but the occurrence quite possible.

5.14. Elaphomyres echinatus Vitt.

Distribution in Europe (Fig. 14). Rare. The examined herbarium material provide some localities in northern Italy and in environs of Paris as 


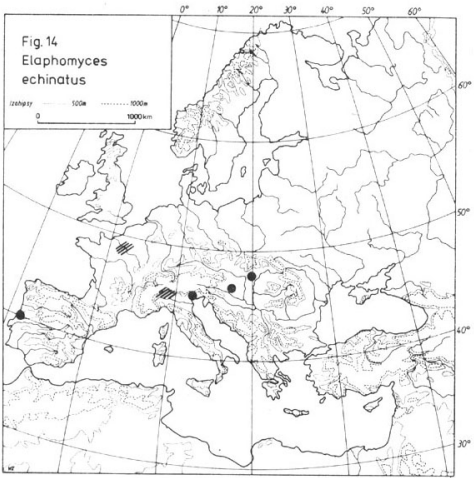

well as single stands in the Ibcrian Peninsula and south from the Carpathian Mtns.

Hypothetical distribution. Wooded hills of S and W Europe in the warmer zone of temperate and submediterranean climate.

Habitat. Under different species of deciduous trecs.

Distribution in Poland. Not yet discovered.

5.15. Elaphomyces cyanosporus Tul.

Distribution in Europe (Fig. 15). Small, scattered localities: in the vicinity of Paris, along the lower Loire river, in northern Italy, in environs of Vienna, and in Central Poland. 


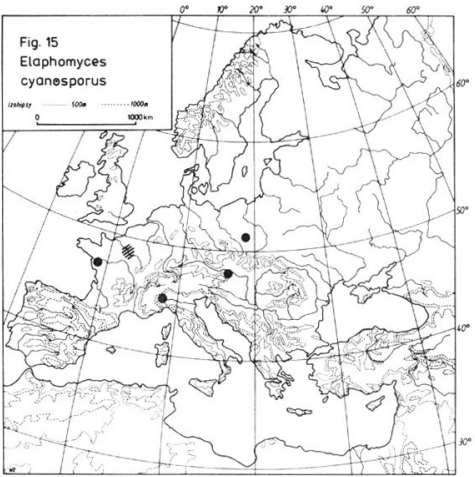

Hypothetical distribution. Lowlands of C and W Europe; temperate, maritime and submaritime climate zones of deciduous forests.

Habitat. Under Quercus, Castanea, and Carpinus betulus in recent fertile humus, in strongly shaded sites with little plant cover and small moss turfs.

Distribution in Poland (Fig. 8). Nizina Poludniowowielkopolska - DE 34 f.r. Wola Wydrzyna n. Szczeroów, Ch TC, 08.80 : RO, LOD 21117.

\subsection{Elaphomyces foetidus Vitt.}

The herbarium material very scant, without indicating localities. Ceruti (1960) reports the species from oak forests in Italy. 


\subsection{Elaphomyces persoonii Vitt.}

Distribution in Europe (Fig. 16). Rare; few localities known south from the Alps, in Appenines, and nearby Paris. In Tulasne \& Tulasne (1851) one read that the species had been reported by Fries from Scania, but this is least probable and not confirmed.

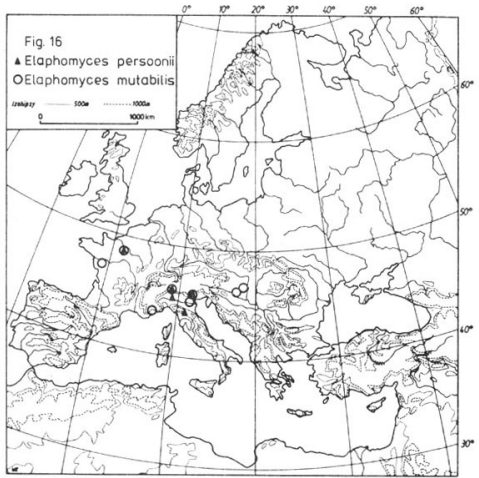

Hypothetical distribution. S and W Europe in the warm zone of temperate and submediterranean climate, in lowlands and low mountain sites.

Habitat. Oak forests, in soils rich in humus.

Distribution in Poland. Not occurring. 
5.18. Elaphomyces mutabilis Vitt.

Distribution in Europe (Fig. 16). On the basis of herbarium material, some localities ascertained in France, Hungary, and northern Italy. Appearing rarely. The data from Germany (Hessc 1894) require to be confirmed by herbarium material.

Hypothetical distribution. Southern, stenothermal species; probably distributed as E. echinatus.

Habitat. Under oaks, chestnuts, birches, and pines.

Distribution in Poland. Not yet discovered.

\subsection{Elaphomyces citrinus Vitt.}

Distribution in Europe (Fig. 17). On the basis of herbarium material, some localities ascertained in northern Italy and southern Switzerland.

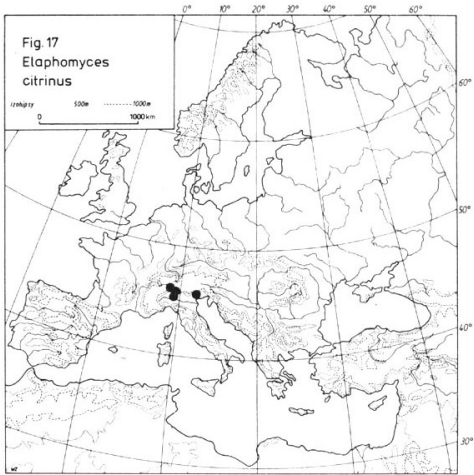


Hypothetical distribution. Southern and, probably, Western Europe in the submediterranean climate zone and the warmer zone of temperate climate.

Habitat. Oak forests.

Distribution in Poland. Not yet discovered.

5.20. Elaphomyces strialosporus Kers

Distribution in Europe. Known from only one locality in Norway: Aker n. Gaustad in the vicinity of Oslo, and one locality in Sweden; originally described by Kers (1980).

Habitat. Under Corylus avellana.

Distribution in Poland. Not yet discovered.

\section{CHOROLOGY OF CENOCOCCUM GEOPHILUM FR. (DEUTEROMYCETES)}

Distribution in Europe. Herbarium material in the form of sclerotia is scant and comes from a few localitics. This does not mean that the species is rare or of limited range. According to the author's investigations their sclerotia can often be found within a quite wide changeability range of habitat conditions (Lawrynowicz 1983).

Hypothetical distribution. Probably entire Europe; the range is as large as that of Elaphomyces muricatus.

Habitat. Woods, peatbogs, moors: in the fresh humus layer; sometimes also cultivated areas.

Distribution in Poland (Fig. 18). Common throughout the whole country in lowlands, uplands and mountains. Most often in recent coniferous forests.

Localities in Poland. Pobrzeże Baftyckie - AB 23 WoNP Wiselka, Fs Qrp Cb, 06.77: ML, LOD 21088-90; - AB 93 Puszcza Bukowa Res. Bukowe Zdroje, Fs, 06.74: Mt, LOD 21091-92. Pojezierze Poludniowopomorskie - CB 44 Bory Tucholskie Konigort, Qrp Cb Tt. 08.74: Mł, LOD 21087; - CB 55 Zukowo, Ps, 07.73: Mt, LOD 21124. Pojezierze Chełmińsko-Dobrzyńskie - DE 95 Ciche n. Zbiczno, Ps, 07.82: RO, LOD 20920. Pradolina Toruńsko-Eberswaldzka - DC 96 Lucien n. Gostynin, Ps, 07.79: RO. LOD 21068; - DC 97 Łack, Ps Pa Qre TC. 07.79: RO, LOD 21040. Pojezierze Wielko polskic - DD 02 frr. Rogoźno, Qrp Cb Pt Ca, 09.79: RO, LOD 21131. Nizina Poludniowowiel. kopolska - CD 79 Sokolöw n. Kożminek, Aps Rp Qrp Fs, 10.78: RO \& Mk, LOD 21065 \& 21085, - Goszczanów, Qrp, 10.78: Mt, LOD 21024; - CD 99 Jasionna n. Blaszki, Qrp Ps Cb: KM, LOD 20796; - CE 09 Kliczków Forests (Lasy Kliczkowskie) n. Jarozłaj \& Sundaly, Ps Qrp, 08.79: KM, LOD 21032; - CE 28 Res. Ryśs n. Lututów, Fs Cb $A a P a, 07$ i 10.79: ML LOD 21022-23: - CE 36 Biadaszki n. Opatów, Qrp Fs, 01.80: RO, 21064: - DD 14 f.r. Ostrowy n. Kutno, Qrp Cb, 06.79: MŁ, LOD 20806 \& 21067; - DD 53 Gostków n. Poddẹbice, Ps Qrp Pa, 04.75: Mh, LOD 21016; - DD 60 Linne n. Dobra, Ps Qrp Pa, 04.75: Mk, LOD 21125 ; - DD 62 Zakrzew n. Poddębice, Qrp Pt, 05.80: KM, LOD 21018 ; - DD 75 kódź Park Ludowy, Qrp Bpp Cb. 10,78 \& 0682: ML, KM \& MK, LOD 20792-93 \& 21002; - DD \&2 f.r. 


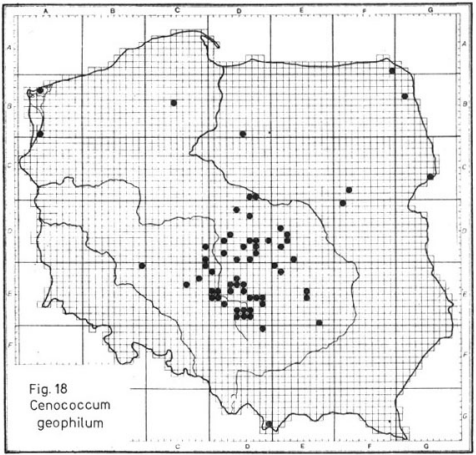

Wojslawice n. Szadek, Aa, 10.81: MK \& KM, LOD 21025; - DD 94 fr. Ostrów n. Lask, Ps, 06.79: RO, LOD 21017; - DE 10 Res. Nowa Wies, Fs, 1080: ML, LOD 21026; - DE 24 Kluki. Ps, 08.78: RO, LOD 21044: - DE 33 f.r. Stróbta n. Szczerców, Ps Bpp Pa, 08.78: ML. LOD 21047-49; - DE 34 i.r. Wola Wydrzyna, Cb Qrp Bpp Ps, 09 \& 10.79, 08.80: ML KM \& RO. LOD 20802, 21027-28 \& 21037; - DE 35 Huba Ruszezyńska n. Szezcroów, Orp Ps cl., 08.78: ML \& RO. LOD 21050-51. Obnizenie Milicko-Glogowskie - BE 09 Niezgoda $n$. Żmigród, Qrp, 06.82, E. Wiącek, LOD 20822. Nizina Środkowomazowiecka - DD 26 f.s. Mnich n. Oporow, $\mathrm{Cb}$ Ps, 05.79: RO, LOD 21069; - ED 41 Puszeza Bolimowska fr. Bolimów, Qup Ps Bpp, 05.82: MK \& KM, LOD 20807-12; - ED 52 f.r. Puszoa Mariańska, Qrp Ps. 09.79, 05 \& 06.82: ML KM \& MK, LOD 20813.21 \& 21059-61: - FC 82 Zgrzebichy n. Stoczek Węrowski, Cb, 10.79: ML 21084: - FD 01 Kąty Borucze n. Mińsk Mazowiecki, Ps Bpp, 10.79: ML LOD 21066. Wzniesienia Południowomazowieckic - DD 66 Lódż Las Lagiewniki, $\mathrm{Pa}, 05.80$ : KM, LOD 21003; - DD $67 \mathrm{fr}$. Janinów n. Brzeziny, Qrp Fs, 10.78: Mt. LOD 21012-13; - DD 69 Res. Zimna Woda n. Rogów, Qrp Ps Cb. 05.74: RO, LOD 21015 ; - DD 79 fr. Wiączyń n. Nowosolna, Fs, 08.75: KM. LOD 20795: - DD 87 Justynów n. Łódz, Qrp Fs Bpp Ps, 10.79: Mt, LOD 21004-05, - Janówka n. Justynów, Fs Qrp. 08.82: 
L. Samosiej, LOD 21006:- DD 96 Res. Wolborka n, Lodz, Ps, 09.76: Mh, LOD 21007, Tuszyn, Ps, 05.77: M. Borowska, LOD 21008, - Res. Molenda, Qrp Ps, 08.74 \& 10.79: Mł, LOD 21009-11, - Modlica, Ch, Ps, Pa, Qrp, 03.80: Mt, LOD 21127, - Szczukwin, Ps, 09.82: W. Malinski, LOD 21014; - ED 62 Res. Babsk, Orp Cb, $07 \& 08.79,04,05 \& 09.80,08.81$ : KM, LOD 20804-05 \& 21054-58; - ED 71 Podlas n. Rawa Mazowiecka, Bpp. 08.81: RO, LOD 21062; - ED 93 Żardki n. Odזzywót, Qrp, 08.79: KM. LOD 21045; - EE 00 Borki n. Spala, Cb. 06.74: ML, LOD 21039. Wyżyna Woźnicko-Wieluniska - DE 40 fr. Mierzycc, Ps Orp $\mathrm{Pa}, 06.79$ \& 08.82: RO \& MK, LOD 20797-800 \& 21030-31; - DE 41 f.r. Nizankowice, Orp Ps, 05 \& $08.82:$ MK, LOD 20801 \& 21029; - DE 43 Res. Murowaniec n. Pajeccno, Aa, 04.79: RO. LOD 21052; - DE 50 Zalecze Male, Aa Ps QrP Pa, 07 \& 08.79: ML \& RO, LOD 20803 \& 21019-2I, - Springs of Revelation (Źródla Objauienia) FI Cisowa, Pa, 07.79: RO, LOD 21053; - DE 51 Giętkowizna n. Parzymiechy, Pa, 04.75; MŁ, LOD 21033; - DE 62 Miedzno n. Klobuck, Qrp Ps, 09.79: MŁ. LOD 21086 . Wyżna Krakowsko-Częstochow . ska - DE 74 Jask rów, Qrp Fs Bpp, 10.79: Mt, LOD 21077: - DE 84 Mirów - Hektary, Qrp Ps, 08.81: RO, LOD 21082; - DE 85 Smiertny Dąb FI Potok Zloty, Ps, 04.75: Mt, LOD 21128, - Lipnik n. Zuraw, Ps Pa Pt, 10.78: MŁ, LOD 21070; - DF 08 Otudza FI Szczekociny, Qrp Ps, 09.82: MK, LOD 21075; - DE 45 Hulanka n. Dobryszyce, Qrp Pa, 09.78 : RO, LOD 21083. Wyzyna Przedborska - DE 56 Res. Jasieri, Ps Pa Bpp, 06 \& 07.75: ML, 20794 \& 21041; - DE 57 fr. Modrzewszeryzna n. Kobiele Wielkie, Ps Qrp Pa, 09.78: RO, LOD 21038; - DE $58 \mathrm{Mt}$. Cheimo, Qrp Cb Pt Pa, 08.79: ML LOD 21080-81: - DE 68 Res. Dębowiec, TC Qrp Cb Tc, 06.79: Mt, LOD 21042; - DE 75 Skrzydlów, Qrp Ps Cb Bpp Fs, 06 \& 07.79. 04.80: ML, LOD 21034-36; - DE 76 f.r. Lipie n. Swįta Anna, Ps Bpp, 08.78: ML, LOD 21046: - DE 86 f.r. Wielki Las n. Swicta Anna, TC Cb Qrp Bpp, 10.79: Mh LOD 21078; - f.r. Knieja, Ps Bpp, 10.79: ML, LOD 21079; - DF 08 f.r. Gąszcze FI Szczekociny, Ps, 09.82: ML \& MK, LOD 21074; - EE 11 Januszewice, PaQ, 08.74: RO, LOD 21063, - Prymusowa Wola, Pa Qrp, 08.73: Mt, LOD 21129. Wyzyna Kielecko-Sandomierska - EE 45 Res. Cisowy Majdów. Ps Pa Aa Bpp. 06.79: MŁ LOD 21073; - EE 55 Res. Świnia Góra, Qrp, 06.74: ML, LOD 21076; - EE 97 Raków - Hańcza, Qrp Ps Pa, 07.77: MŁ. LOD 21071.72. Beskidy Zachodnie - DG 19 Rabka Hill (Wzgórze) Bania, $P a, 11.79:$ ML, LOD 21130. Tatry - DG 59 Koscieliska Dale (Dolina K.) mouth of the Smytna Dale, Pa, 10.79: ML, LOD 21097. Pojezierze Litewskie - FA 99 Rybalnia, $P a$ Ps, 08,79: RO, LOD 21096; - GB 31 Res. Starożyn, TC Qrp Pa, 08.79: RO, LOD 21094, - Res. Mały Borek, Ps Pa, 08.79: RO, LOD 21095. Nizina Pótnocnopodlaska - GC 55 BiNP, TC, 05.78: ML, LOD 21126: GC 65 Czerlonka, Qrp, 05.80: RO, LOD 21093.

The author thanks to Professors: Alina Skirgichlo and Romuald Olaczek for help in preparing the maps and manuscript. and to David Pegler (Kew) for improving the English translation.

\section{REFERENCES}

Ainsworth G. C, 1983, Ainsworth and Bisby's Dictionary of Fungi. 7th ed. Kew.

Babos M., 1981, Cheiramyces tenosus and Terfezia terfezioides in Hungary. A fehér szarvasgom. ba és a mohoki szarvasgomba elterjedése Magyarosrágon. Mikol. Kiszl. 1-2: 47-56.

Berkeley M. J. Broome C. E, 1844, Notices of British Fungi. Ann. Mag. Nat. Hist. (1) 13: $340-360$.

Bis by G., 1933. The distribution of fungi as compared with that of phanerogams. Amer. J. Bot. $20(4): 147-192$.

Bucholtz F, 1902, Beitrăge zur Morphologie und Systematik der Hypogăen (Tuberaceen und 
Gasteromyceten etc.) nebst Beschreibung aller bis jetzt in Russiand angetroffenen Arten. Riga.

Ceruti A. 1960, Elaphomycetales and Therales [In] J. Bresadola, Iconographia Mycologica 28. Suppl. II. Trento.

Chatin A., 1892 La Truffe. Botanique de la Truffe et des plantes truificeres. Paris.

Delmas J. 1978, Tuber spp. [In:] S. T. Chang. W. A. Hayes (Editors). The Biology and Cultivation of Edible Mushrooms. Acad. Press, New York-San Francisco-London, pp. 645-681.

De Vries G. A., 1971, De Fungi van Nederland. 3. Hypogaea. Wetenschappelijke Mededelingen Konink lijke Nederlandse Natuurhistorische Vereniging 88: 1-64.

Eckblad F. E., 1954, Studies in the hypogean fungi of Norway. I. Endogone and Tuberales. Nytt. Mag. Bot. 3: 35-41.

Eckblad F. E., 1962 Studies in the hypogean fungi of Norway. II. Revision of the genus Elaphomyces. Ibid. 9: 199-210.

Eichler B., 1904, Drugi przyczynek do flory grzybów okolie Międzyrzeca. Pam. Fizjogr. 18: 1. 31.

Fischer E., 1897, Tuheraceae und Hemiasceae. In: L Rabenhorst: Kryptogamen-Flora von Deutschland, Osterreich und der Schweiz. 2. Aufl. Bd. I. Pilzc. V. Abt. Ascomycetes. S. Ordn. Tuberaceat, Leiprig.

Gross G., 1977. Rund um dic "Deutsche weisse Truffel", Choiromyces meandriformis Vitt. Z. f. Pilzk. 43: 85-96.

Hawker L. E., 1954, Bratish Hypogeous Fungi. Phil. Trans. Roy, Soc. London 237: 429-546. Hesse R., 1894, Die Hypogaeen Deutschlands. Die Tuberaceen und Elaphomyceten. II. Halle Saake,

Hollós L, 1911, Magyarorság foldalatti gombái, sarvasgombafćtri. Fungi Hypogaei Hun* gariae. Budapest.

Kers L. E. 1978, Elaphomyes maculatus found in Sweden. Bot. Notiser 131: 419-422.

Kers L. E., 1979a, Geneu werrucosa funnen i Sverige (Genea terrucosa found in Sweden). Svensk. Bot. Tidskr. 72: 309-311.

Kers L E, 1979b, Elaphemyces aculeat us funnen i Sverige (Elaphomyces aculeatus found in Sweden). Ibid. 73: 123.126.

Kers L. E. 1980, A new species of Elaphemyces Nees ex Fr. subgen. Malacoderma Vitt. Bot. Notiser $133(2): 149-153$.

Kers L. E. 1981. On the identity of Elaphomyces thigmesus Hesse (Ascomycetes). Nord. J. Bot. 1: $795-800$.

Kers L. E, 1983, Elaphemyces anthracinus och E. lecellle i Norden' (Elaphomyces anthracimas and E. Lereillei in Norden). Svensk Bot. Tidskr. 77: 43-56.

K napp A., 1950-52. Die europäischen Hypogacen-Gauungen und ihre Gattungstypen. Schweiz. Zeitschr. Pilzk, (1950) 28 (3): 29-42, 28 (7) $101-116,28$ (10): 153-179: (1951) 29 (4): 65-92. 29 (7): $133-148:(1952) 30(3): 33-43,30(6): 81-92$.

Kondracki J., 1977. Regiony fvyczno-geografiezne Polski. Warsawa.

Kreisel H. Dörfelt H. Benkert D, 1980, Karten zur Pflanzen-verbreitung in der DDR. Hercynia 17 (3): 233-291.

Lange L. 1974, The distribution of Macromycetes in Europe. Dansk Bot. Arkiv 30 (1): 1-105. Lange M. 1956. Danish Hypogeous Macromyceles. Ibid. 16 (1): 1 -84.

Lawrynowicz M. 1970, Występowanie grzybów z rodzajöw Elaphomyces i Cordyceps w wojewódzlwie lódzkim. Zeszyty nauk owe Uniwersytetu Lódzkiego 36: 89-93.

Lawrynowiez M, 1983, Cenococcum graniforme w Polsee. Acta Mycol, 19 (1): 31-40.

Lawrynowicz M. 1988, Elaphomycelales, Tuberales, [In:] Flora Polska. PWN, Warseawa -Kraków. 
Lubelska B., 1953, O wystcpowanu trufli (Tuber Mich. i Choiromyces Vitt.) w Polsce. Fragm.

Flor. Geobot. 1: $87-96$.

Nicolas J. J., 1973, La Trufa. Bot. Est. Central Ecologia 3: 3-28.

Railviir A. G. 1964, Geograficheskoe rasprost ranenie geterobazidiainykh griboy, Eesti NSV

Tead. Akad. Toim. 13: 106-122

Schrocter J. 1908, Pibe. In: Cohn's Kryptogamen-Flora von Schlesien. 3 (2). Breslau.

Schwärzcl C. 1967-69, Beitray zur Hypogeenflota des Kantons Basel-Stadt und seiner

Umgebung. Schweiz. Zcitschr. Pilzk. (1967) 45 (9): 129-141; (1968) 46 (12): 189-198: (1969)

47 (8): $149-161$.

Skirgietto A., 1976, Materiaty do poznania roemieszczenia geograficzncgo grzybów wyżsych

w Europie. V. Acta Mycol. 12 (2): 155-189.

Skirgietto A, Wosiriska A., 1963, O rormieszcreniu jeleniaków (Elaphomyces) w Polsce.

Monogr. Bot 15: 361-371.

Szemere L. 1965, Die unterirdischen Pilze des Karpatenbeckens, Budapest.

Teodorowicz F- 1928, Ober massenhaftes Vorkommen der Geopora Michaelis (Fisch.)

Löchertrüfel in Polen. Z Pilzk, 15: 79-80.

Teodorowicz F., 1933, Grzyby Zachodnicj i Poludniowej Polski. Wydawn. Okreg. Kom.

Ochr. Przyr. na Wielkop. Poznan.

Teodorowicz F., 1936, Grzyby wyzsze polskiego wybrzėa. Tow. Nauk, w Toruniu. Bad. Przyr. Pomorskic, Torun.

Trampler T., Smykala J., Bosiak A, 1980, Instrukcja urządzania lasu. I. PWRjL, Watszawa.

Tulasne L. R., Tulasne C, 1851. Fungi Hypogaei. Histoire et monographie des champignons hypoges. Paris.

Vasileva L. N, 1967, Agarikovye ili shlapochnye griby (por, Agaricales) Primorskogo kraja. Avtograf dis Erevan.

Vasileva L. N. 1973, Agarikovye shlapochnye griby Primorskogo kraja. Izd. „Nauka“, Leningrad,

Vasilk ov B. P.. 1955, Ochcrk gcograficheskogo rasprostranenia shlapochnyk h gribov v SSSR.

Izd. Akad. Nauk SSSR, Moskva-Leningrad.

Vittadini $\mathrm{C}_{2}, 1831$, Monographia Tuberacearum. Mediolani.

Vittadini C., 1842, Monographia Lycoperdincertum. Mediolani. Also published in Mem. Reale Acad. Sci. Torino (2) 5 .

Wasser S. P. 1980. Flora gribov Ukrainy, Agarikovye griby. Izd. "Naukova Dumka”, Kiev. Zajac A. 1978, Zatórenia metodyczne, Atlasil rozmieszczenia roslin naczyniowych w Polsce". Wiad. Bot. 22 (3) 145-155.

Zobel J. B., 1854, In: A. C. J. Corda. Icones Fungorum hucusque cognitorum. Pragae. 